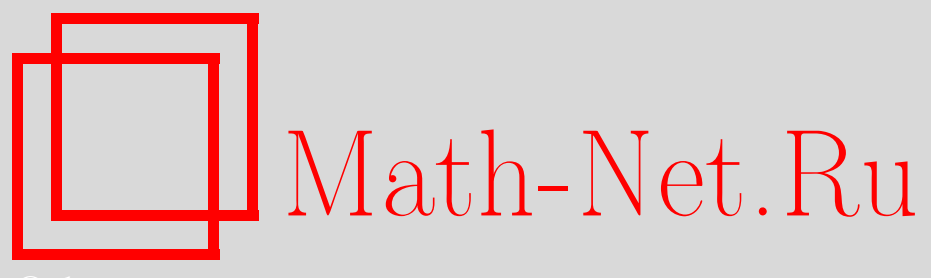

П. Г. Гриневич, А. Е. Миронов, С. П. Новиков, О нерелятивистском двумерном чисто магнитном суперсимметричном операторе Паули, УМН, 2015, том 70, выпуск 2, 109-140

DOI: https://doi.org/10.4213/rm9650

Использование Общероссийского математического портала Math-Net.Ru подразумевает, что вы прочитали и согласны с пользовательским соглашением http://www . mathnet.ru/rus/agreement

Параметры загрузки:

IP : 3.85 .7 .115

26 апреля 2023 г., 13:33:40

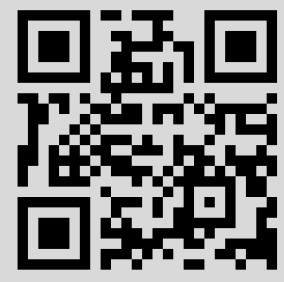




\title{
О нерелятивистском двумерном чисто магнитном суперсимметричном операторе Паули
}

\author{
П. Г. Гриневич, А. Е. Миронов, С. П. Новиков
}

В статье полное многообразие собственных функций основного состояния для чисто магнитного 2D-оператора Паули рассматривается как побочный продукт новой редукции, найденной авторами несколько лет назад для алгебро-геометрических обратных спектральных данных (т. е. римановых поверхностей и дивизоров). Эта редукция ассоциирована с $(2+1)$-солитонной иерархией, содержащей 2D-аналог известной "системы Бюргерса". Эта статья содержит также изложение предыдущих работ, выполненных с 1980 г., включая первые топологические идеи в пространстве квазиимпульсов. Мы также приводим новые результаты, посвященные самосопряженным граничным задачам для оператора Паули. Нулевой 2D-уровень "неспектральных" функций Блоха-Флоке дает дискретные точки дополнительного спектра аналогично "граничным состояниям" конечнозонных 1D-потенциалов в зонах.

Библиография: 35 названий.

Ключевые слова: магнитный оператор Паули, алгебро-геометрические решения, основное состояние, уровни Ландау, краевые задачи.

DOI: $10.4213 / \mathrm{rm} 9650$

СОДЕРЖАНИЕ

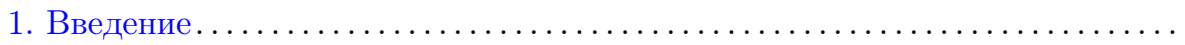

2. История: основные состояния, магнитно-блоховские функции. Тополо-

гия типичных законов дисперсии .................... 110

3. Суперсимметрия и преобразования Лапласа .................... 114

4. Алгебро-геометрические скалярные операторы Шрёдингера. Проблема

редукции ......................................... 117

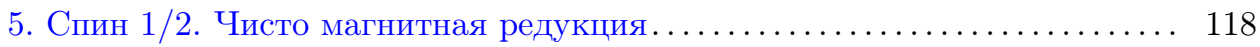

6. Случай рода нуль. Типы вещественных решений .............. 120

Исследование первого и третьего авторов выполнено при поддержке РФФИ (грант № 13-01-12469-офи-м2) и программы "Ведущие научные школы" (грант НШ-4833.2014.1). Первый автор поддержан также программой Президиума РАН "Фундаментальные проблемы нелинейной динамики". Исследование второго автора выполнено за счет гранта Российского научного фонда (проект 14-11-00441).

Цикл предыдущих работ авторов по данной теме можно найти на персональной странице С.П. Новикова www.mi.ras.ru/ snovikov, см. Scientific Publications, статьи номер 177, 178, 179, 181, 183. 
7. Решения рода 1. $\delta$-образные члены ....................... 123

8. Распространение результатов на бесконечные тригонометрические ряды

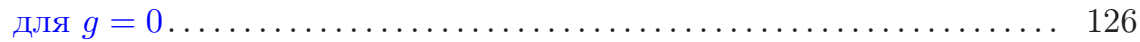

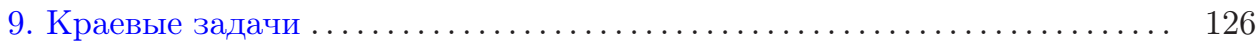

9.1. Граничные условия для операторов Паули без смешивания ком-

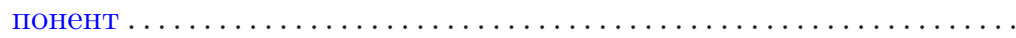

9.2. Граничные условия для операторов Паули со смешиванием компонент . . . . . . . . . . . . . . . . . . . . . . . . . 128

10. Блоховские функции вне спектра как решения краевых задач ...... 129

Список литературы . . . . . . . . . . . . . . . . . . . . . . 138

\section{1. Введение}

В общем случае движение заряженной частицы во внешнем электромагнитном поле описывается полным 4D-уравнением Дирака (для спина 1/2). Паули вывел красивую нерелятивистскую аппроксимацию $L^{P}$ этого оператора (см. [1; $\S 33])$. Мы рассмотрим специальный 2D-случай, когда электрическое поле равно нулю, в котором раскрывается замечательная внутренняя симметрия этого оператора. Теория основных состояний для него началась в 1979-1880 гг. В настоящей работе мы описываем ее объединение с алгебро-геометрической спектральной теорией для одного уровня энергии периодического скалярного оператора Шрёдингера и соответствующей теорией солитонных нелинейных систем. Ключевую роль здесь играет двумерный аналог иерархии Бюргерса. Члены типа Ааронова-Бома в магнитном поле вида "квантованной" дельта-функции играют важную роль в нашей конструкции. Эта статья развивает результаты, опубликованные в [2]-[6]. Мы обращаем особое внимание на самосопряженные граничные условия, позволяющие выявить роль блоховских функций с неунитарными мультипликаторами и другими сингулярностями в теории квантовых состояний для областей с границей. Нами найдены обширные многообразия таких решений. Подобные идеи были известны для $D=1$ с 1974 г. в теории конечнозонных операторов Шрёдингера, где нули блоховских функций вели к интепретации этих функций как "приграничных состояний", но при $D>1$ аналогичная интерпретация никогда не обсуждалась. Какие самосопряженные граничные условия кроме условий Дирихле реализуются в квантовой механике реальных систем? Мы не знаем ответа на этот вопрос.

\section{2. История: основные состояния, магнитно-блоховские функции. Топология типичных законов дисперсии}

В 1979-1980 гг. три группы авторов изучали основной уровень, используя "свойство факторизации" чисто магнитного 2D-оператора Паули, записанного в лоренцевой калибровке $A_{1 x}+A_{2 y}=0$, где $A_{1}=i \Phi_{y}, A_{2}=-i \Phi_{x}$ :

- Аврон-Зайлер (АЗ) [7];

- Ааронов-Кашер (AK) [8];

- Дубровин-Новиков (ДН) [9], [10]. 
Оператор Паули является двухкомпонентным оператором второго порядка шрёдингерского типа. Мы используем специальную систему единиц такую, что

$$
L^{P}=L^{+} \oplus L^{-}, \quad-L^{ \pm}=\left(\partial_{x}+i \Phi_{y}\right)^{2}+\left(\partial_{y}-i \Phi_{x}\right)^{2} \pm \Delta \Phi .
$$

Оператор действует на пространстве вектор-функций $\Psi=\left(\Psi^{+}, \Psi^{-}\right)$.

Мы предполагаем, что заряд равен 1. Следующее формальное наблюдение является очень важным.

Лемма 1. Пусть $Q=\partial_{z}-A_{z}, Q^{+}=-\left(\partial_{\bar{z}}+A_{\bar{z}}\right)$, где $\partial_{z}=\partial=\partial_{x}-i \partial_{y}$, $A_{z}=A_{1}-i A_{2}$. Скалярные операторь $L^{ \pm}$раскладываются на множстели: $L^{+}=Q Q^{+}, L^{-}=Q^{+} Q$, и для магнитного поля имеется формула $B=\Delta \Phi$.

Доказательство заключается в прямой подстановке.

Наиболее интересными классами магнитных полей являются АК и ДН. Были получены следующие результаты (их доказательство приведено в теореме 1 ниже).

1. АК: бъстро убъвающие поля,

$$
|[B]|=\left|\iint_{\mathbb{R}^{2}} B d x d y\right|<\infty .
$$

Основные состояния образуют конечномерное пространство размерности $m \in \mathbb{Z}$, $m \leqslant[B] /(2 \pi)<m+1$.

2. ДН: произвольные периодические поля с иелым потоком через элементарную ячейку:

$$
\frac{1}{2 \pi} \iint_{\text {cell }} B d x d y=m \in \mathbb{Z} .
$$

Основные состояния порождают бесконечномерное подпространство в гильбертовом пространстве $L_{2}\left(\mathbb{R}^{2}\right)$, изоморфное уровню Ландау. Так называемые "магнитно-блоховские состояния", собственные для так называемых "магнитных трансляций" (см. лемму 2), образуют многообразие, изоморфное полному пространству векторного расслоения над двумерным тором магнитных квазиимпульсов (см. (2), (3)). Проекция на базу в точности совпадает с так называемым "магнитным квазиимпульсом". Первый класс Черна этого расслоения равен базисному классу когомологий в группе $H^{2}\left(T^{2}, \mathbb{Z}\right)$, слой изоморфен $\mathbb{C}^{m}$. Мы приведем соответствующие формулы в конце раздела 2.

Опыт работы в дифференциальной топологии после Х. Уитни, Л. С. Понтрягина и Р. Тома привел С. П. Новикова около 1980 г. к идее изучать "общие" операторы, удовлетворяющие требованиям трансверсальности для спектра, чтобы определить их топологические инварианты. Дело в том, что отнюдь не случайные классы операторов Шрёдингера, возникающие в квантовой теории частиц и кристаллов, являются периодическими или быстроубывающими. Физические спектры характеризуются не абстрактными множествами и мерами, а гладкими или комплексными многообразиями, для которых естественна идеология дифференциальной топологии. Это позволило выявить топологические инварианты операторов в пространстве квазиимпульсов (квазимоментов). В частности, в 1980-1981 гг. С. П. Новиковым и А. С. Лысковой (см. [11]-[14]) были 
открыты и изучены числа Черна трансверсальных законов дисперсии, каждый из которых представляет собой комплексное одномерное векторное расслоение над тором $T^{2}$ в случае общего положения в двумерной задаче. Дело в том, что оператор Шрёдингера в периодических полях (магнитное поле должно иметь целочисленный поток сквозь элементарную ячейку) представляет собой семейство комплексных эрмитовых эллиптических операторов над тором в случае общего положения, где спектр дискретен и никогда не является кратным в двумерном случае общего положения, так как условие кратности имеет коразмерность 3 среди комплексных эрмитовых операторов. Это обстоятельство было предметом дискуссии Дж. фон Неймана и Ю. Вигнера еще в 1930-х годах, но топологические следствия впервые обсуждались С.П. Новиковым около 1980 г. Тем самым, каждая мода определяет векторное 1-расслоение над тором. Было установлено, что первый класс Черна может быть любым. Этот результат легко следует из теории возмущений однородного магнитного поля с потоком сквозь элементарную ячейку, равным $m>1$. Совокупность магнитно-блоховских функций одного уровня Ландау образует векторное расслоение с классом Черна $c_{1} \in H^{2}\left(T^{2}, \mathbb{Z}\right)$, базисным в этой группе. После возмущения это расслоение распадается на $m$ одномерных расслоений с классами Черна $c_{1}^{j}$, где $\sum_{j} c_{1}^{j}=c_{1}$. А. С. Лыскова показала, что в остальном слагаемые произвольны и любая топологическая возможность реализуется. В типичных однопараметрических семействах двумерных операторов Шрёдингера возникают изолированные точки, где законы дисперсии сталкиваются и один передает другому единицу класса Черна. Несколькими годами позже эти идеи были переоткрыты физиками группы Д. Таулеса (D. Thouless) в процессе поиска объяснения известного "целочисленного квантового эффекта Холла", открытого экспериментально в то время [15], [16].

Теорема 1. В случаях АК и ДН все основные состояния являются инстантонами принадлежащими только одному спиновому сектору. Это означает следующее.

(а) Они удовлетворяют уравнениям первого порядка: $Q^{+} \psi=0$ в секторе $L^{+}$в случае $[B]>0$ и $Q \psi=0$ в секторе $L^{-}$в случае $[B]<0$. Это является простым прототипом уравнения автодуалъности.

(b) Они принадлежат гильбертову пространству $L_{2}\left(\mathbb{R}^{2}\right)$.

ДокАЗАТЕЛьство. 1. Случай АК. Пусть $\Psi$ является нулевой модой для оператора $L^{+} \Psi=0$ и $\Psi \in L_{2}$. Стандартный "инстантонный аргумент" для основного состояния таков: $0=\left\langle Q Q^{+} \Psi, \Psi\right\rangle=\left\langle Q^{+} \Psi, Q^{+} \Psi\right\rangle$. Таким образом, $Q^{+} \Psi=0$. Каждое решение $\Psi \in L_{2}$ этого уравнения, интегрируемое с квадратом, определяет нулевую моду оператора $L^{+}$, и обратно. Таким образом, наш результат будет вытекать из существования решений из пространства Гильберта $L_{2}\left(\mathbb{R}^{2}\right)$. Пусть $0<[B]<\infty, m \leqslant[B] /(2 \pi)<m+1$. Мы ищем $\Psi$ в виде произведения двух сомножителей:

$$
\Psi_{l}=P_{l}(z) \exp \{-R(x, y)\}
$$


где $P_{l}, l=0, \ldots, m$, есть произвольный голоморфный полином, а $R(x, y)$ есть специальное решение уравнения $\Delta R=B$ при $[B]>0$ :

$$
R(x, y)=-\frac{1}{4 \pi i} \iint_{\mathbb{R}^{2}} \ln |z-w| d w \wedge d \bar{w} .
$$

Рост $R$ очевидно зависит от величины магнитного потока $[B]$ :

$$
\iint_{\mathbb{R}^{2}}\left|\Psi_{l}\right|^{2} d x \wedge d y<\infty
$$

при $l=0, \ldots, m-1$. Таким образом, мы нашли все основные состояния в случае AK. Во втором секторе $L^{-}$не существует квадратично интегрируемых решений.

2. Случай ДН. Решения здесь также могут быть найдены только в одном спиновом секторе. Как и выше, мы предполагаем, что магнитный поток через элементарную ячейку является положительным целым числом $m \in \mathbb{Z}_{+}$. Мы рассмотрим прямоугольную решетку с периодами $2 \omega \in \mathbb{R}, 2 i \omega^{\prime} \in i \mathbb{R}$. Определим $R$ формулой

$$
R=-\frac{1}{4 \pi i} \iint_{K} \ln (|\sigma(z-w)|) B(w, \bar{w}) d w \wedge d \bar{w},
$$

где $\Delta R=B, K$ - элементарная ячейка. Мы ищем решения, представимые в виде

$$
Q^{+} \Psi=0, \quad \Psi=\exp \{-R(x, y)\}\left[e^{a z} \prod_{j=1, \ldots, m} \sigma\left(z-a_{j}\right)\right] \lambda,
$$

где $\lambda \neq 0$ - произвольное число.

Лемма 2. Решения, представимые в виде (1), являются магнитно-блоховскими функииями, т.е. собственными векторами магнитных трансляuиц̆ $T_{j}^{*}, j=1,2$ :

$$
\begin{aligned}
& T_{1}^{*} \Psi(x, y)=\Psi(x+2 \omega, y) e^{-i f_{1}(x, y)}=\varkappa_{1} \Psi(x, y), \\
& T_{2}^{*} \Psi(x, y)=\Psi\left(x, y+2 \omega^{\prime}\right) e^{-i f_{2}(x, y)}=\varkappa_{2} \Psi(x, y) .
\end{aligned}
$$

Вектор-потенциал, как и выше, выбран в лоренцевой калибровке $A=\left(i \Phi_{y},-i \Phi_{x}\right)$ для $B=\Delta \Phi$ и $\Phi=-R$. По определению

$$
\begin{aligned}
& A(x+2 \omega, y)=A(x, y)+i\left(f_{1 x}, f_{1 y}\right), \\
& A\left(x, y+2 \omega^{\prime}\right)=A(x, y)+i\left(f_{2 x}, f_{2 y}\right) .
\end{aligned}
$$

ДокАЗАТЕЛьство. Мы используем стандартные свойства преобразований

$$
\sigma(w+2 \omega)=-e^{-2 \eta(w+\omega)} \sigma(w), \quad \sigma\left(w+2 i \omega^{\prime}\right)=-e^{-2 i \eta^{\prime}\left(w+i \omega^{\prime}\right)} \sigma(w),
$$

где $\zeta(w)=\sigma_{w} / \sigma, \eta=\zeta(\omega), i \eta^{\prime}=\zeta\left(i \omega^{\prime}\right)$ для прямоугольной решетки.

Наша теорема немедленно вытекает из этих свойств. 
После вычислений мы приходим к следующей формуле для параметров, отвечающих унитарным мультипликаторам $\left|\varkappa_{1}\right|=\left|\varkappa_{2}\right|=1$ :

$$
\begin{aligned}
& \operatorname{Re} a=\operatorname{Re}\left\{\frac{\eta_{1}}{\omega}\left[2 \sum_{j} a_{j}-\frac{1}{\pi} \iint_{K} z B(x, y) d x d y\right]\right\}, \\
& \operatorname{Im} a=\operatorname{Im}\left\{\frac{\eta^{\prime}}{\omega^{\prime}}\left[2 \sum_{j} a_{j}-\frac{1}{\pi} \iint_{K} z B(x, y) d x d y\right]\right\} .
\end{aligned}
$$

По определению компонент квазиимпульсов $p_{1}, p_{2}$ имеем:

$$
e^{2 i p_{1} \omega}=\varkappa_{1}, \quad e^{2 i p_{2} \omega^{\prime}}=\varkappa_{2} .
$$

Они имеют следующий вид, вытекающий из формул для магнитно-блоховских функций:

$$
\begin{aligned}
& p_{1}+\frac{m \pi}{2 \omega}=\operatorname{Im}\left(a-\frac{\eta}{\omega} \sum_{j} a_{j}\right), \\
& p_{2}+\frac{m \pi}{2 \omega^{\prime}}=\operatorname{Re}\left(a-\frac{\eta^{\prime}}{\omega^{\prime}} \sum_{j} a_{j}\right) .
\end{aligned}
$$

Отображение квазиимпульсов корректно определено на пространстве параметров, описывающих состояния с унитарными мультипликаторами. Оно выглядит следующим образом:

$$
p_{1}+i p_{2}=p\left(a_{1}, \ldots, a_{m}\right)=-\frac{2 \pi i}{|K|} \sum_{j} a_{j}+\text { const },
$$

где $|K|=4 \omega \omega^{\prime}-$ площадь элементарной ячейки $K$. Образом является тор $T^{2}$, слоем является комплексное пространство $\mathbb{C}^{m}$.

Элементарные аргументы из [9], [10] показывают, что эти семейства описывают все собственные состояния оператора $L^{P}$ в окрестности основного уровня $\epsilon=0$. Это следует из свойств эллиптического оператора $L^{P}$ с фиксированными унитарными мультипликаторами. Его ядро является в точности $m$-мерным, так как индекс оператора $Q^{+}: \Psi^{+} \rightarrow \Psi^{-}$равен $m$ и сопряженный оператор $Q: \Psi^{-} \rightarrow \Psi^{+}$не имеет ядра. Сингулярности не появляются в этом семействе ни для каких точек тора квазимоментов (т.е. ни для каких унитарных мультипликаторов). Таким образом, мы заключаем, что существует конечный ненулевой интервал $\Delta$, отделяющий нулевой уровень от всех других уровней оператора $L^{P}$.

\section{3. Суперсимметрия и преобразования Лапласа}

Оператор $S=Q^{+}: \Psi^{+} \rightarrow \Psi^{-}, S\left(\Psi^{-}\right)=0$ называется суперсимметрией для $L^{P}$. Здесь $S^{2}=0, S L^{P}=L^{P} S, S^{*} L^{P}=L^{P} S^{*}$. "Сопряженным" оператором суперсимметрии является $S^{*}=Q: \Psi^{-} \rightarrow \Psi^{+}, S^{*}\left(\Psi^{+}\right)=0$. Имеем $S S^{*}+S^{*} S=L^{P}$. 
Последнее равенство влечет, что все высшие уровни являются двукратно вырожденными (основной уровень является бесконечно вырожденным). Оператор суперсимметрии обсуждался в физической литературе 1980-х годов (см., например, [17]).

На самом деле мы встречаемся здесь с частным случаем так называемого преобразования Лапласа, которое было известно с XVIII в. для скалярных 2D-операторов Шрёдингера. Каждый гиперболический оператор второго порядка $L=\partial_{x} \partial_{y}+A \partial_{x}+B \partial_{y}+V$ может быть представлен в виде

$$
L=\left(\partial_{x}+B\right)\left(\partial_{y}+A\right)+W=Q Q^{\prime}+W .
$$

Определим

$$
L \rightarrow \widetilde{L}=W Q^{\prime} W^{-1} Q+W, \quad \Psi \rightarrow \widetilde{\Psi}=Q^{\prime} \Psi .
$$

Можно показать, что из $L \Psi=0$ следует $\widetilde{L} \widetilde{\Psi}=0$ и

$$
\widetilde{L}=\left(\partial_{y}+A-\frac{W_{y}}{W}\right)\left(\partial_{x}+B\right)+W=\left(\partial_{x}+B\right)\left(\partial_{y}+A-\frac{W_{y}}{W}\right)+\widetilde{W},
$$

где $\widetilde{W}=W-A_{x}+B_{y}+(\ln W)_{x y}$.

Мы будем иметь дело с эллиптическим случаем, который важен для применений в квантовой механике. Заменим $\partial_{x}$ на $\partial=\partial_{z}$ и $\partial_{y}$ на $\bar{\partial}$. Таким образом,

$$
L=Q Q^{+}+W \rightarrow \widetilde{L}=W Q^{+} W^{-1} Q+W \quad \text { и } \quad \Psi \rightarrow Q^{+} \Psi=\widetilde{\Psi},
$$

где $Q=\partial+A, Q^{+}=-\bar{\partial}+\bar{A}$. Для специального случая факторизуемых операторов $W=$ const это преобразование действует на всем спектре оператора $L$. Физики отождествляют это с "суперсимметрией" для оператора Паули: $L \Psi=\epsilon \Psi$ влечет $\widetilde{L} \widetilde{\Psi}=\epsilon \widetilde{\Psi}$ для $\widetilde{\Psi}=Q^{+} \Psi$.

Калибровочными инвариантами оператора $L=Q Q^{+}+W$ являются магнитное поле $2 B=A_{\bar{z}}-\bar{A}_{z}$ и потенциал $W=e^{f}$. Удобно выписывать преобразование Лапласа только в терминах инвариантов:

$$
\widetilde{W}=W+\widetilde{B}, \quad \widetilde{B}=B+\frac{1}{2} \Delta \ln W .
$$

Было замечено, что вся бесконечная цепочка преобразований Лапласа

$$
W_{n}=e^{f_{n}}=\widetilde{W}_{n-1}, \quad B_{n}=\widetilde{B}_{n-1}
$$

эквивалентна "2D-цепочке Тоды”. Это наблюдение (конечно, в гиперболическом случае) восходит к XIX в. Оно было сделано в школе Дарбу (см. [18]). Эта школа начала также рассматривать циклические цепочки преобразований Лапласа и указала, что они ведут к нескольким вполне интегрируемым системам включая sin-Гордон. Эти работы содержат только формальные вычисления, посвященные гиперболическому случаю (см. исторические обсуждения в [19]). Мы можем исключить магнитное поле из соотношения $W_{n+1}-W_{n}=B_{n+1}$. Это приводит к уравнению

$$
\frac{1}{2} \Delta f_{n}=e^{f_{n+1}}-2 e^{f_{n}}+e^{f_{n-1}} .
$$


Подстановка $f_{n}=g_{n}-g_{n-1}$ приводит это уравнение к двумерной цепочке Тода. В работе С. П. Новикова и А. П. Веселова (1997, [19]) циклические цепочки были рассмотрены в эллиптическом случае, где глобальные ограничения играют фундаментальную роль: для гладких эллиптических двоякопериодических операторов в [19] было доказано, что соответствующие операторы Шрёдингеpa $L$ всегда являются алгебро-геометрическими, т. е. множество гладких комплексных решений Блоха-Флоке уравнения $L \Psi=0$ образует алгебраическую кривую (т. е. риманову поверхность конечного рода).

Доказательство этой теоремы основывается на том, что решения циклической цепочки удовлетворяют эллиптическому уравнению в частных производных. Несингулярные периодические решения, тем самым, образуют конечномерные многообразия. В полной аналогии с аргументами работ С. П. Новикова и Б. А. Дубровина 1974-1975 гг. [20], отсюда сразу следует, что эти решения алгебро-геометрические, так как циклическая цепочка - это вполне интегрируемая система в смысле современной теории (представление Лакса и т.д.). Высшие потоки линейно зависимы на конечномерном многообразии. Это и приводит к конечнозонности (алгебро-геометричности) решений. Подобный аргумент, по-видимому, впервые был замечен Н. Хитчиным [21] для гармонических торов в $\mathbb{R}^{3}$ и использован У. Пинкалем и И. Стерлингом при изучении поверхностей постоянной средней кривизны торической топологии в $\mathbb{R}^{3}$ (см. обзор А. И. Бобенко [22]).

Впрочем, эта теория содержит только топологически тривиальные операторы с двоякопериодическими коэффициентами и нулевым потоком магнитного поля через элементарную ячейку. Для того чтобы включить топологически нетривиальные операторы, в [19] был рассмотрен другой тип цепочек преобразований Лапласа. Пусть $L_{0} \rightarrow L_{1} \rightarrow \cdots \rightarrow L_{n}$ - цепочка преобразований Лапласа такая, что крайние операторы $L_{0}, L_{n}$ раскладываются на множители с точностью до константы:

$$
\begin{gathered}
L_{n}=C_{n}+Q_{n} Q_{n}^{+}, \quad L_{0}=Q_{0} Q_{0}^{+}, \\
C_{n}=n\left[B_{0}\right], \quad\left[B_{0}\right]=2 \pi m>0, \quad m \in \mathbb{Z},
\end{gathered}
$$

где $[B]$ - поток магнитного поля $B$ сквозь элементарную ячейку решетки. Основной уровень является здесь многократно вырожденным для гладкого двоякопериодического магнитного поля $B_{0}$. При $n=2$ были найдены нетривиальные случаи такие, что поле $B_{n}$ также гладко. Поэтому один высший уровень энергии $\epsilon=n\left[B_{0}\right]$ (происходящий из основного уровня для оператора $Q_{n} Q_{n}^{+}$, переброшенного с помощью цепочки Лапласа к оператору $\left.L_{0}\right)$ также бесконечно вырожден. Для доказательства бесконечной кратности вырождения достаточно найти гладкие двоякопериодические решения уравнения

$$
\frac{1}{4} \Delta u=-e^{u}+\left[B_{0}\right], \quad e^{u}=W_{0}=B_{0} .
$$

Таких решений существует много. Для длин цепочки более двух $(n>2)$ мы нетривиальных решений не знаем.

Согласно нашей гипотезе, не более двух уровней могут быть бесконечнократно вырождены, исключая случай оператора Ландау в однородном магнитном поле, и один из них, скорее всего, основной. 


\section{4. Алгебро-геометрические скалярные операторы Шрёдингера. Проблема редукции}

Boпрос: связана ли теория основного состояния чисто магнитного оператора Паули с алгебро-геометрической теорией скалярного периодического 2D-оператора Шрёдингера на одном уровне энергии и солитонными 2D-иерархиями?

Напомним историю этого объекта.

Алгебро-геометрическая теория скалярных 2D-операторов Шрёдингера на одном уровне энергии и соответствующих солитонных иерархий была начата в 1976 г. С. В. Манаковым [23] и Б. А. Дубровиным, И. М. Кричевером, С. П. Новиковым [24].

Магнитное поле всегда топологически тривиально в этой теории (т. е. коэффициенты вектор-потенциалов двоякопериодичны).

Идея использования системы

$$
\frac{d L}{d t}=(L H-H L)+f L
$$

вместо обыкновенной пары Лакса была предложена в [23]. Нетривиальный пример с операторами $L, H$ порядка 2 демонстрировал, что новый подход содержателен. В течение долгого времени эта специфическая система не исследовалась. При решении обратной задачи для $L$ на одном уровне энергии в [24] была введена “двухточечная скалярная функция Бейкера-Ахиезера". Это позволило построить всю иерархию высших систем, ассоциированную с первоначальной. В частности, вся иерархия совершенно отлична от случая Кадомцева-Петвиашвили: она зависит от двух бесконечных множеств времен $t_{j}^{\prime}, t_{j}^{\prime \prime}$, отвечающих двум "бесконечным" точкам. Риманова поверхность здесь является семейством всех несингулярных комплексных функций Блоха-Флоке на одном выбранном уровне энергии $L \psi=0$ ("комплексная кривая Ферми"). В алгебро-геометрическом случае она имеет конечный род. На этой алгебраической кривой Ферми выбраны две "бесконечные" точки и локальные параметры в них. Следует задать также дивизор полюсов $D$ степени, равной роду. Этот набор данных определяет и блоховскую функцию, и оператор $L$ (вообще говоря, комплексный), и всю иерархию нелинейных систем, с ним связанных (его деформаций с помощью троек Манакова - см. выше).

Некоторые редукции в этих уравнениях (т.е. динамически инвариантные подсистемы или подыерархии) активно изучались в 1980-х годах. Несколько авторов нашли такие редукции либо для нелинейных систем, либо для обратных спектральных данных (или данных рассеяния), либо в обоих случаях. Решение этой задачи для обратных спектральных данных более трудно: в частности, оно влечет описание всех редуцированных иерархий. Впрочем, практически существование редукций обнаружить гораздо проще для уравнения, чем для данных обратной задачи.

Наша главная цель здесь - квантовая механика и спектральная теория. Мы используем нелинейные системы в качестве инструмента для этой цели.

В первой работе [24] 1976 г. ее авторы нашли только чисто вещественные редукции спектральных данных.

1. Данные, отвечающие периодическим самосопряженным операторам, были найдены И. В. Чередником в 1980 г. [25]. 
2. Данные, отвечающие операторам $L=-\Delta+U(x, y)$ (с нулевым магнитным полем), были найдены в 1984 г. (см. [26]-[29]). Необходимость условий Новикова-Веселова пока не доказана строго, хотя это несомненно верно.

Расширение этих результатов на быстро убывающие потенциалы было исследовано в работах С. В. Манакова, П. Г. Гриневича, Р. Г. Новикова и С. П. Новикова в конце 1980-х годов (см. [30]-[32]). И. М. Кричевер в [33] доказал, что каждый гладкий периодический 2D-потенциал может быть аппроксимирован алгебро-геометрическими операторами.

\section{5. Спин $1 / 2$. Чисто магнитная редукция}

Как найти алгебро-геометрические данные для редукции, приводящей к факторизуемым операторам? Эта задача недавно была решена авторами (см. [2]-[6]).

Решение задачи редукции. Рассмотрим первоначальную систему Манакова $L_{t}=[H, L]+f L$, где оба оператора $L, H$ являются операторами второго порядка:

$$
L=\partial_{x} \partial_{y}+G \partial_{y}+S, \quad H=\Delta+F \partial_{y}+A .
$$

Получившаяся система имеет вид

$$
\begin{aligned}
G_{t} & =G_{x x}-G_{y y}+\left(\frac{F^{2}}{4}\right)_{x}-\left(G^{2}\right)_{x}-A_{x}+2 S_{y}, \\
S_{t} & =S_{y y}-S_{x x}-2(G S)_{x}+(F S)_{y}
\end{aligned}
$$

с дифференциальными ограничениями

$$
F_{x}=2 G_{y}, \quad A_{y}=2 S_{x}, \quad f=2 G_{x}-F_{y} .
$$

Б. Г. Конопельченко уже указывал в 1988 г. (см. [34]), что редукция $S=0$ является динамически инвариантной для этой системы. Она выглядит как 2D-аналог знаменитой системы Бюргерса.

Мы верим в следующий неформальный принцип: каждая динамически инвариантная редукция вполне интегрируемой солитонной системы может быть эффективно описана в терминах (спектральных) данных рассеяния и римановых поверхностей.

Как описать эту специфическую редукцию для обратных спектральных данных? Это возможно? Делая замену $x, y \rightarrow z, \bar{z}$, мы приходим к наиболее интересным для нас эллиптическим операторам.

Наш недавний результат [2]-[6] описывает соответствующую обратную задачу для оператора $L \mathbf{c} S=0$. Возьмем приводимую риманову поверхность (комплексную кривую Ферми), состоящую из двух несингулярных компонент $\Gamma=\Gamma^{\prime} \cup \Gamma^{\prime \prime}$ родов $g^{\prime}, g^{\prime \prime}$. Компоненты пересекают друг друга по $P_{j}=Q_{j}, P_{j} \in \Gamma^{\prime \prime}, Q_{j} \in \Gamma^{\prime}, j=0,1, \ldots, l$ (см. рис. 1 для самосопряженного эллиптического случая, где $\left.g^{\prime}=g^{\prime \prime}\right)$. Возьмем бесконечности $\infty_{1} \in \Gamma^{\prime}, \infty_{2} \in \Gamma^{\prime \prime}$ с локальными параметрами $\left(k^{\prime}\right)^{-1},\left(k^{\prime \prime}\right)^{-1}$. Построим функцию $\psi=\left(\psi^{\prime}, \psi^{\prime \prime}\right)$ с асимптотикой $\psi^{\prime} \sim c(x, y) e^{k^{\prime} \bar{z}}\left(1+O\left(\left(k^{\prime}\right)^{-1}\right)\right), \psi^{\prime \prime}=e^{k^{\prime \prime} z}\left(1+O\left(\left(k^{\prime \prime}\right)^{-1}\right)\right)$ и дивизоры полюсов $D^{\prime}, D^{\prime \prime}$ степеней $g^{\prime}+l, g^{\prime \prime}$, не содержащие бесконечностей и точек 
пересечения. В гиперболическом случае возьмем переменные $x, y$ вместо $z, \bar{z}$. Временная динамика может быть добавлена стандартным образом:

$$
\begin{aligned}
& \psi^{\prime}=\exp \left\{k^{\prime} \bar{z}+\sum_{s>1}\left(k^{\prime}\right)^{s} t_{s}^{\prime}\right\}\left(c+O\left(\left(k^{\prime}\right)^{-1}\right)\right), \\
& \psi^{\prime \prime}=\exp \left\{k^{\prime \prime} z+\sum_{s>1}\left(k^{\prime \prime}\right)^{s} t_{s}^{\prime \prime}\right\}\left(1+O\left(\left(k^{\prime \prime}\right)^{-1}\right)\right) .
\end{aligned}
$$

Условие пересечения имеет вид

$$
\psi^{\prime}\left(Q_{j}\right)=\psi^{\prime \prime}\left(P_{j}\right), \quad j=0,1, \ldots, l .
$$

Теорема 2. Такие данные задают двухточечную функиию Бейкера-Ахиезера $\psi=\left(\psi^{\prime}, \psi^{\prime \prime}\right)$ на приводимой поверхности $\Gamma=\Gamma^{\prime} \cup \Gamma^{\prime \prime}$ и скалярный оператор $L^{\prime}=\Delta+G \partial_{\bar{z}}$ maкой, чmo $S=0 u L^{\prime} \psi^{\prime}=L^{\prime} \psi^{\prime \prime}=0$.

Данные для самосопряженных операторов. Для выделения самосопряженных операторов необходимо обеспечить выполнение равенства $g^{\prime}=g^{\prime \prime}$, а также добавить вырожденное ограничение типа Чередника. Можно сказать, что это ограничение является просто пределом условия Чередника для вырожденной римановой поверхности $\Gamma=\Gamma^{\prime} \cup \Gamma^{\prime \prime}$. Возьмем бесконечные точки $\infty^{\prime} \in \Gamma^{\prime}$ с локальным параметром $\left(k^{\prime}\right)^{-1}$ и $\infty^{\prime \prime} \in \Gamma^{\prime \prime}$ с локальным параметром $\left(k^{\prime \prime}\right)^{-1}$. Антиголоморфное отображение $\tau: \Gamma^{\prime \prime} \rightarrow \Gamma^{\prime}$ должно быть задано так, что $\tau\left(\infty^{\prime \prime}\right)=\infty^{\prime}, \tau^{*}\left(k^{\prime}\right)=\overline{k^{\prime \prime}}$ и $\tau$ переставляет точки пересечения: $\tau\left(P_{j}\right)=Q_{k_{j}}$. Обратное отображение $\Gamma^{\prime} \rightarrow \Gamma^{\prime \prime}$ мы также обозначим через $\tau$, таким образом, $\tau: \Gamma \rightarrow \Gamma, \tau^{2}=1$. Дивизор полюсов $D^{\prime} \in \Gamma^{\prime}$ и $D^{\prime \prime} \in \Gamma^{\prime \prime}$ степеней $\left|D^{\prime}\right|=g^{\prime}+l$ и $\left|D^{\prime \prime}\right|=g^{\prime \prime}$, где $g^{\prime}=g^{\prime \prime}$, не содержит точек пересечения и бесконечностей. Дивизором полюсов на приводимой римановой поверхности Г является $D=D^{\prime} \cup D^{\prime \prime}$. Мы, как обычно, предполагаем, что существует мероморфная 1-форма $\Omega$ с простыми полюсами в точках дивизоров $D, \tau(D)$ и в бесконечных точках $\infty_{1}, \infty_{2}$. Дивизор $(K)$ состоит из нулей голоморфной 1 -формы, символ " " обозначает "линейную эквивалентность" дивизоров:

$$
D+\tau(D) \sim(K)+\infty^{\prime}+\infty^{\prime \prime} .
$$

Теперь пусть наша поверхность будет вырождена. 1-форма $\Omega$ также становится вырожденной. Ее вырождение состоит из двух мероморфных 1-форм $\omega^{\prime}, \omega^{\prime \prime}$ на римановых поверхностях $\Gamma^{\prime}, \Gamma^{\prime \prime}$ с простыми полюсами в точках пересечения $P_{j} \rightarrow \tau\left(Q_{j}\right)$ таких, что $\operatorname{res}_{P_{j}} \omega^{\prime \prime}+\operatorname{res}_{Q_{j}} \omega^{\prime}=0$. Их дивизоры должны удовлетворять следующим условиям:

$$
\begin{aligned}
& \left(\omega^{\prime}\right)=D^{\prime}+\tau\left(D^{\prime \prime}\right)-\infty^{\prime}+\sum_{j} Q_{j}, \\
& \left(\omega^{\prime \prime}\right)=D^{\prime \prime}+\tau\left(D^{\prime}\right)-\infty^{\prime \prime}+\sum_{j} P_{j} .
\end{aligned}
$$

После неунитарного калибровочного преобразования

$$
L^{\prime} \rightarrow L=\frac{1}{\sqrt{c}} L^{\prime} \sqrt{c}, \quad \psi \rightarrow \frac{\psi}{\sqrt{c}}
$$


в $\Gamma^{\prime}, \Gamma^{\prime \prime}$ мы получаем самосопряженный оператор

$$
L=Q Q^{+}=(\partial-A)(-\bar{\partial}-\bar{A}), \quad A=\frac{1}{2} \bar{\partial} \ln c .
$$

Беря $L^{+}=L$ и $L^{-}=Q^{+} Q$, мы построим чисто магнитный оператор Паули

$$
L^{P}=Q Q^{+} \oplus Q^{+} Q
$$

Магнитное поле задается формулой

$$
B=\frac{1}{2} \Delta \ln c
$$

оно вещественно, периодично или квазипериодично и топологически тривиально. Оно несингулярно, если $c \neq 0$, таким образом, в этом случае оператор самосопряжен.

Как найти основные состояния в гильбертовом пространстве? Это очень просто в периодическом несингулярном случае $c \neq 0$. Возьмем $\psi_{0}=$ $c^{1 / 2}$ в первом спиновом секторе $L^{+}$, пользуясь тем, что $Q^{+} \psi_{0}=0$. Возьмем $\phi_{0}=c^{-1 / 2}$ во втором секторе $L^{-}$, пользуясь тем, что $Q \phi_{0}=0$. В случае периодического гладкого вещественного $c \neq 0$ мы имеем в точности две периодические функции основного состояния, сосредоточенные в обоих секторах. Они отвечают дну непрерывного спектра возле основного состояния $\epsilon=0$. В разделах 6-8 мы дадим полное описание всех несингулярных блоховских функций основного состояния. Их связь с магнитно-блоховскими функциями, найденная в [9] в 1980 г. для топологически нетривиального магнитного поля, будет обсуждаться в разделе 7.

\section{6. Случай рода нуль. Типы вещественных решений}

Опишем данные для рода нуль (см. рис. 1).

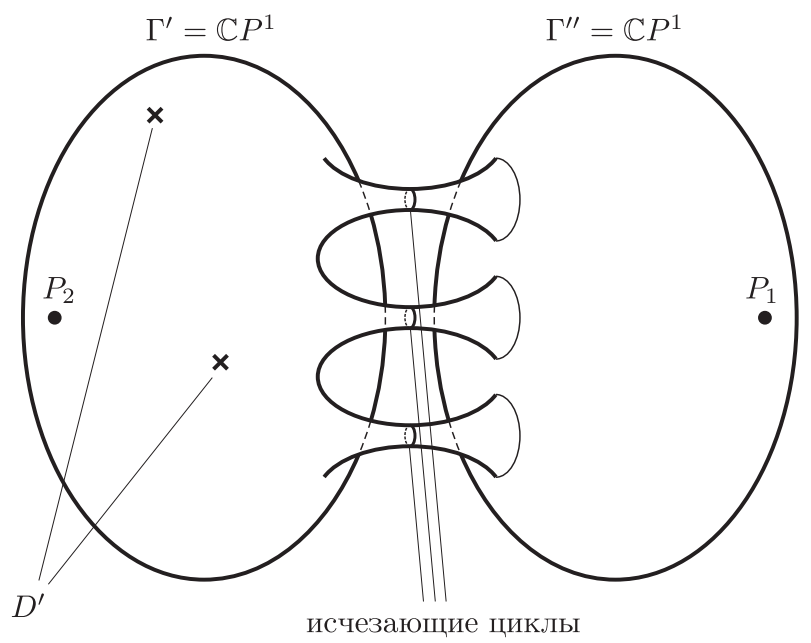

Рис. 1 
Возьмем $l+1$ точку пересечения с координатами $k^{\prime}=k_{s}, k^{\prime \prime}=p_{s}$ в $\Gamma^{\prime}, \Gamma^{\prime \prime}$ и дивизор $D^{\prime}=\left(a_{1}, \ldots, a_{l}\right)$ степени $l$ в $\Gamma^{\prime}$. Эти алгебро-геометрические данные определяют функцию Бейкера-Ахиезера. Имеем

$$
\Psi^{\prime}=e^{k^{\prime} \bar{z}} \frac{w_{0}\left(k^{\prime}\right)^{l}+\cdots+w_{l}}{\left(k^{\prime}-a_{1}\right) \cdots\left(k^{\prime}-a_{l}\right)},\left.\quad \Psi^{\prime}\right|_{k^{\prime}=k_{s}}=e^{p_{s} z} .
$$

Как можно видеть, $c=w_{0}$. Таким образом,

$$
c=\sum_{s=0}^{l} \kappa_{s} e^{W_{s}(z, \bar{z})},
$$

где $W_{s}=p_{s} z-k_{s} \bar{z}$ (см. [3]). В [3] доказана следующая важная теорема.

Теорема 3. Для каждого экспоненииального/тригонометрического полинома с указанного выше вида с произвольным множеством линейных форм $W_{s}$ и комплексных коэффициентов $\kappa_{s}$ существуют алгебро-геометрические данные, отвечающие этой функиии с.

Пусть $W_{s}=\alpha_{s} x+\beta_{s} y,\left(\alpha_{s}, \beta_{s}\right) \in \mathbb{C}_{W}^{2}$. Преобразование $c \rightarrow c^{\prime}=c e^{\gamma+\alpha x+\beta y}$ приводит к калибровочно эквивалентному оператору (с тем же магнитным полем).

\section{Существуют следующие три типа вещественных решений.}

1. Чисто экспоненциальный положительный случай ("поле лампового типа") $\kappa_{s}>0,\left(\alpha_{s}, \beta_{s}\right) \in \mathbb{R}^{2}$.

2. Вещественный периодический тригонометрический случай. Он будет рассмотрен ниже вместе со случаем $g=1$.

3. Смешанный экспоненциальный/тригонометрический случай. Он может быть реализован, только если его “доминирующая часть" принадлежит случаю 1. Мы не будем обсуждать его.

Рассмотрим случай 1. Пусть "тропическая сумма" форм в множестве $\{W\}$ является неотрицательной:

$$
I_{\{W\}}^{\prime}(\phi)=\max _{s}\left(\alpha_{s} \cos \phi+\beta_{s} \sin \phi\right) \geqslant 0 .
$$

Тогда $c^{-1 / 2}$ ограничена в $\mathbb{R}^{2}$. Для углов $I_{\{W\}}^{\prime}(\phi)>0$ мы имеем быстрое убывание $c^{-1 / 2} \rightarrow 0, R \rightarrow \infty$. Пусть $I(\phi)=\max \left\{I^{\prime}(\phi), 0\right\}$ (см. рис. 2a).

В каждом классе $c^{\prime} \in c e^{W}, W^{\prime} \in \mathbb{R}_{W}^{2}$ множество представителей $c^{\prime}$ с неотрицательным $I=I_{\left\{W^{\prime}\right\}}^{\prime}(\phi) \geqslant 0$ образует выпуклый многоугольник $\bar{T}_{c}$. Его внутренность $T_{c} \subset \bar{T}_{c}$ состоит из всех $c^{\prime}$ таких, что $I_{\left\{W^{\prime}\right\}}>0$. Открытая часть $T_{c}$ всегда непуста при $l>2$. Множество $\bar{T}_{c}$ непусто при $l>1$ (см. рис. $2 \mathrm{~b}$ при $l=3)$.

Магнитное поле является убывающим при $R \rightarrow \infty$ исключая некоторые выбранные углы, это аналоги ламповых полей для “ламповых потенциалов" Кадомцева-Петвиашвили. Линейный характер суммы (5) в (4) отражает линеаризацию иерархии Бюргерса в терминах переменной $c$. Мы имеем

$$
[B]=\iint_{D_{R}^{2}} B d x d y=-\frac{R}{2} \oint_{S^{1}} I_{\{W\}}(\phi) d \phi+O\left(R^{-1}\right) .
$$




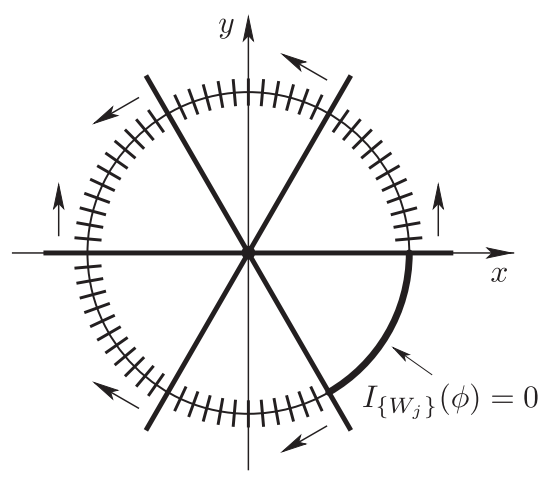

Рис. 2a. $I(\phi)$ для $c=e^{y}+e^{y-2 x}+e^{-y-2 x}$

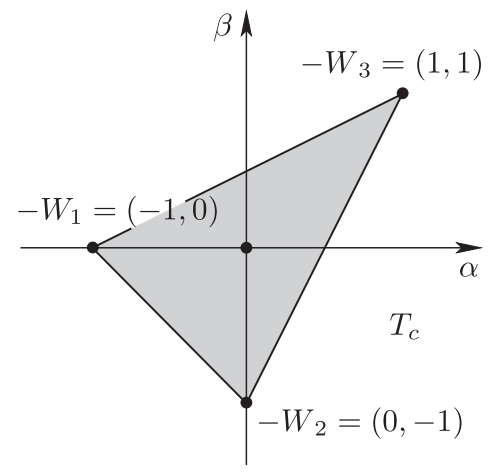

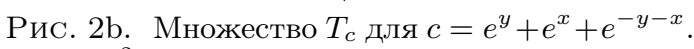

Здесь $\mathbb{R}^{2}=(\alpha, \beta), W_{j}=\alpha_{j} x+\beta_{j} y$.

Все точки в $T_{c}$ определяют основные состояния в гильбертовом пространстве $L_{2}\left(\mathbb{R}^{2}\right)$. Граничные точки определяют дно непрерывного спектра. Интеграл квадрата собственных функций $\left(c^{\prime}\right)^{-1 / 2}$ конечен для $c$, лежащих строго внутри множества

$$
T_{c}: \int_{\mathbb{R}^{2}} \frac{d x d y}{c}<\infty .
$$

Это легко проверить, например, для рассмотренного выше случая $c=e^{y}+e^{x}+$ $e^{-y-x}$, где интеграл легко исследуется.

Периодическая задача. Пусть решетка в $\mathbb{R}^{2}$ прямоугольна и $z=x+i y$. Для каждой вещественной периодической функции $c$ мы можем определить целое семейство "возможных" мероморфных блоховских функций

$$
\psi_{\mathrm{ext}, \pm}^{\prime \prime}=f(z)(\sqrt{c})^{ \pm} e^{u z-\zeta(p) z} \frac{\sigma(z+p+R)}{\sigma(z+R)},
$$

где $f(z)$ - произвольная эллиптическая функция.

Имеем $Q^{+} \psi_{\text {ext,- }}^{\prime \prime}=0$ для $L=L^{+}=Q Q^{+}$. Для антиголоморфных функций мы получаем $Q \psi_{\mathrm{ext},+}^{\prime \prime}=0$ при $L^{-}=Q^{+} Q$. 
Пусть $c \neq 0$. Нам нужны только несингулярные функции, таким образом, нашим многообразием является $u \in \mathbb{C} P^{1}=\Gamma^{\prime \prime}$ с функцией $\psi_{+}^{\prime \prime}=e^{u z} \sqrt{c}$ (или $\left.e^{u \bar{z}} \sqrt{c}\right)$.

Пусть $c$ имеет изотропный нуль. Мы имеем большее семейство несингулярных (или “слабо сингулярных") $\psi_{+}^{\prime \prime}$-блоховских функций, так как функция $\sqrt{c} / \sigma(z+R)$ слабо сингулярна и блоховская функция может входить в спектр. Таким образом, всем многообразием является $M^{2}=\mathbb{C} P^{1} \times \Gamma$, где $\Gamma-$ эллиптическая кривая. Если $c$ есть тригонометрический полином, то $1 / c-$ не тригонометрический полином. Мы имеем пару функций: $c$ для $L^{+}$и $c^{\prime}=1 / c$ для $L^{-}$. Таким образом, мы должны найти $\psi^{\prime}$ для всех гладких вещественных периодических функций $c^{\prime}$. Это будет сделано ниже, после разбора рода 1.

\section{7. Решения рода $1 . \delta$-образные члены (рис. 3 )}

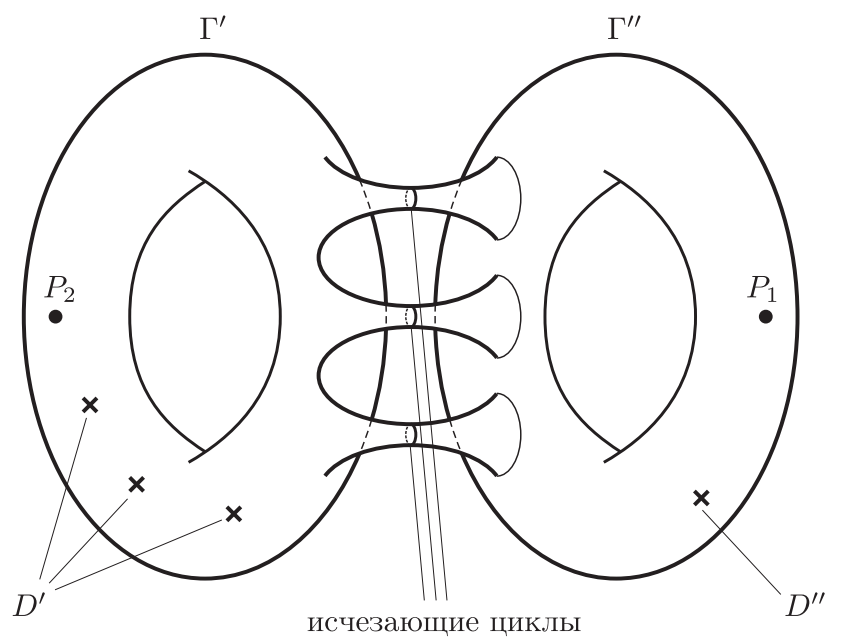

Рис. 3

Возьмем эллиптическую кривую $\Gamma^{\prime}=\Gamma^{\prime \prime}=\mathbb{C} / \Lambda$ с евклидовыми локальными параметрами $k, p$ (точка 0 является "бесконечностью"), имеющую периоды $2 \omega \in \mathbb{R}, 2 i \omega^{\prime} \in i \mathbb{R}$. Зададим $n+1$ точку пересечения $Q_{0}, \ldots, Q_{n} \in \Gamma^{\prime}$ и $R_{0}, \ldots, R_{n} \in \Gamma^{\prime \prime}$. Дивизоры $D^{\prime}=\left(P_{1}, \ldots, P_{n}\right), D^{\prime \prime}=P$ имеют степени $n+1,1$ соответственно. Имеем

$$
\psi^{\prime}=e^{-\bar{z} \zeta(k)} \frac{\prod_{s} \sigma\left(k-Q_{s}\right)}{\prod_{l} \sigma\left(k+P_{l}\right)}\left(\sum_{j} w_{j} \frac{\sigma\left(k+\bar{z}+\widetilde{P}+\widetilde{Q}-Q_{j}\right)}{\sigma\left(k-Q_{j}\right)}\right)
$$

(здесь $\widetilde{P}=P_{1}+\cdots+P_{n}, \widetilde{Q}=Q_{0}+\cdots+Q_{n}$, сумма как в $\left.\mathbb{C}\right)$ и

$$
\psi^{\prime \prime}=e^{-z \zeta(p)} \frac{\sigma(p+z+P)}{\sigma(z+P) \sigma(p+P)}, \quad \psi^{\prime}\left(Q_{s}\right)=\psi^{\prime \prime}\left(R_{s}\right) .
$$

Все сингулярности величины $c$ исчезают после умножения

$$
\widetilde{c}=c \sigma(\bar{z}+\widetilde{Q}+\widetilde{P}) \sigma(z+P) .
$$


Возьмем $n=1, Q_{1}=-Q_{0}, R_{0}=Q_{1}, R_{1}=Q_{0}$, где $Q_{0}$ есть решение уравнения $\omega \zeta\left(Q_{0}\right)=\eta_{1} Q_{0}$. В этом случае имеем $P=\widetilde{Q}+\widetilde{P}$, т. е. $-(1 / 2) \Delta|\sigma|^{2}=-2 \pi \delta(z)$.

Таким образом, вывод, основанный на случае $g=1$, является следующим: наименьшие вещественные сингулярные данные отвечают периодическому несингулярному магнитному полю $\widetilde{B}=-(1 / 2) \Delta \widetilde{c}$ с магнитным потоком, равным одной квантовой единице. Изначальное алгебро-геометрическое топологически тривиальное магнитное поле $B=(1 / 2) \Delta c$, полученное по нашим спектральным данным, всегда является сингулярным при $g=1$; оно имеет нулевой магнитный поток через элементарную ячейку и квантованную $\delta$-сингулярность на фоне несингулярного поля $\widetilde{B}$, сосредоточенного в точке $P$. Таким образом, это поле отвечает ситуации "Ааронова-Бома".

При $g>1$ число квантованных $\delta$-функций равно $k>1$. Мы можем получить несингулярные алгебро-геометрические операторы только из рода 0 . Обе компоненты приводимой римановой поверхности $\Gamma=\Gamma^{\prime \prime} \cup \Gamma^{\prime}$ представляются в виде $k$-листного накрытия над эллиптической кривой $\Gamma^{\prime \prime} \rightarrow \Gamma_{0}$ при $g>1$, так же как это было в работах И. М. Кричевера, посвященных эллиптическому уравнению Кадомцева-Петвиашвили (см. [35]).

Важный вывод: сопоставление с [9] показывает, что квантованный $\delta$-поток не влияет на спектр вблизи нулевого уровня.

Комплексные многообразия Блоха-Флоке (состоящие из несингулярных или слабо сингулярных блоховских функций, допускаемых спектральной задачей) на уровне $\epsilon=0$ и при $g=1$ суть

$$
M=M^{2}
$$

с функцией

$$
\begin{gathered}
\psi_{\mathrm{ext},-}^{\prime \prime}=\frac{1}{\sqrt{c}}\left(e^{u z} \cdot e^{-\zeta(p) z} \frac{\sigma(z+p+R)}{\sigma(z+R)}\right), \\
L^{+} \psi_{\mathrm{ext}}^{\prime \prime}=L^{+} \psi^{\prime}=0 .
\end{gathered}
$$

Отметим, что обе функции $\psi^{\prime}, \psi^{\prime \prime}$ изначально сингулярны при $g=1$. Наш следующий шаг - редуцировать оператор к самосопряженному виду. Для этого мы должны умножить обе функции $\psi^{\prime}, \psi^{\prime \prime}$ на $1 / \sqrt{c}$. Начнем с $\psi^{\prime \prime}$. Функция $\psi_{\text {ext }}^{\prime \prime} \sqrt{c}$ является "несингулярной", т. е. она достаточно слабо сингулярна, чтобы войти в $L_{2}\left(\mathbb{R}^{2}\right)$-спектр для оператора $L$ с квантованным членом Ааронова-Бома. После сингулярных унитарных калибровочных преобразований $L \rightarrow \widetilde{L}$ все сингулярности этой функции исчезают. Таким образом, она входит в гладкий спектр оператора $\widetilde{L}$ с магнитным полем $\widetilde{B}$, у которого сингулярность исчезает. Теперь рассмотрим $\psi^{\prime}$. Функция $\psi^{\prime} / c$ несингулярна, но функция $\psi^{\prime} / \sqrt{c}$ является сингулярной. После сингулярного унитарного калибровочного преобразования она переходит в функцию $\widetilde{\psi^{\prime}} / \sqrt{c}$ с простым полюсом. Справедливо уравнение

$$
\widetilde{L}\left(\sqrt{\frac{\sigma}{\bar{\sigma}}} \frac{\psi^{\prime}}{\sqrt{c}}\right)=0 .
$$

Таким образом, мы заключаем, что семейство $\widetilde{\psi}^{\prime} / \sqrt{c}$ магнитных блоховских функций для оператора $\widetilde{L}$ с несингулярным топологически нетривиальным магнитным полем имеет полюс по переменным $(x, y)$. 
Функция $\psi^{\prime} / c$ является несингулярной. Она удовлетворяет уравнению

$$
L_{0}\left(\frac{\psi^{\prime}}{c}\right)=0
$$

во всех несингулярных точках, где $L_{0}=\partial\left(\bar{\partial}+\Phi_{z}\right)$. В то же время мы имеем $\Phi_{z} \sim 1 / \bar{z}$ в окрестности сингулярности, все остальные члены являются гладкими. Таким образом, функция $f=\left(\bar{\partial}+\Phi_{z}\right)\left(\psi^{\prime} / c\right), c=e^{\Phi}$, является почти всюду антиголоморфной. В то же время в точке $P$, где имеется сингулярность, $f$ имеет вид $f \sim m(k) / \bar{z}$. Поэтому около этой точки $\psi^{\prime} / c \sim m(k)+\bar{z} O(1)$. Мы заключаем, что $L_{0}\left(\psi^{\prime} / c\right)=m(k) \delta_{P}(x, y)$, так как $\partial(1 / \bar{z})=$ (const) $\delta$.

Пересмотрим теперь случай $g=0$, сопоставляя его с $g=1$. При $c \neq 0$ и $g=0$ блоховское многообразие является объединением двух компонент $\Gamma^{\prime \prime} \cup \Gamma^{\prime}$, каждая из которых есть $\mathbb{C} P^{1}$. Пусть $c$ имеет изолированный нуль (минимум), который является изотропным. Магнитное поле становится сингулярным с $\delta$-особенностью. Расширенная инстантонная блоховская функция $\psi_{\mathrm{ext},+}^{\prime \prime}$ становится слабо сингулярной и способной сохранить спектр оператора $L^{+}$. Это зависит от точек комплексного двумерного многообразия $M^{2}$, и все возможные значения комплексных мультипликаторов

$$
M^{2} \rightarrow\left(\varkappa_{1}, \varkappa_{2}\right) \in \mathbb{C}^{*} \times \mathbb{C}^{*}
$$

представлены в этом семействе. Закон дисперсии для энергии здесь вырожден: $\epsilon: M^{2} \rightarrow 0$.

Имеем $M^{2}=\mathbb{C} P^{1} \times \Gamma_{0}$ (здесь $\Gamma_{0}-$ эллиптическая кривая) и

$$
\psi_{\text {ext },+}^{\prime \prime}=\operatorname{const}(u) e^{p \bar{z}-\zeta(u) \bar{z}} \frac{\sigma(\bar{z}+u)}{\sigma(\bar{z})} \sqrt{c} .
$$

Ее сингулярность имеет вид $\sqrt{\bar{\sigma} / \sigma}$, так как $c$ имеет решетку двойных нулей вида $|\sigma|^{2}$. Сделаем сингулярное калибровочное преобразование

$$
\psi_{\text {ext },+}^{\prime \prime} \rightarrow \sqrt{\frac{\sigma}{\bar{\sigma}}} \psi_{\text {ext },+}^{\prime \prime}
$$

(здесь $\sigma$ зависит от $\bar{z}$ ). Эта функция преобразуется в несингулярную магнитную блоховскую функцию оператора $L^{P}$ с топологически нетривиальным магнитным полем $\widetilde{B}$. Магнитные блоховские мультипликаторы после преобразования становятся стандартными блоховскими мультипликаторами.

Наш вывод состоит в следующем: периодический случай $g=1$ дает результат, аналогичный случаю $g=0$ со специально выбранной функцией $c$ : $c$ имеет изолированный изотропный нуль, с точностью до перестановки секторов \pm (т. е. $z$ и $\bar{z}$ ). Большее число $k \geqslant 1$ изотропных нулей для $g=0$ приводит к семейству "высокого ранга" слабо сингулярных блоховских функций инстантонного типа $M^{k+1}$. Удаляя $\delta$-сингулярности сингулярными калибровочными преобразованиями, мы получаем гладкое магнитное блоховское многообразие $M^{k+1}$, отвечающее периодическому магнитному полю с высоким потоком и аналогичное построенному в [9]. 


\section{8. Распространение результатов на бесконечные тригонометрические ряды для $g=0$}

Мы знаем, что алгебро-геометрический случай отвечает случаю тригонометрических полиномов. Возьмем прямоугольную решетку в плоскости $x, y$. Выполнено следующее соотношение:

$$
Q^{+} \psi^{\prime}=M(k) \sqrt{c} e^{\bar{z} k} .
$$

Теперь выберем нормализацию $\psi^{\prime}$ такую, что $M(k)=k$.

Воспользуемся формулой

$$
\psi^{\prime}=k \sum_{j} \frac{\kappa_{j} e^{p_{j} z-k_{j} \bar{z}}}{k-k_{j}} e^{k \bar{z}} .
$$

Мы имеем дело с новой нормализацией: здесь $k_{j}$ являются элементами решетки.

Как следствие,

$$
\sum_{j} \kappa_{j} e^{p_{j} z-k_{j} \bar{z}}=c .
$$

Применим этот результат к бесконечному ряду $c \rightarrow c^{\prime}=1 / c$. Это дает нам функцию $\psi_{-}^{\prime}$ для второй компоненты $L^{-}$оператора Паули.

\section{9. Краевые задачи}

ЗАДАчА. Компонента $\Gamma^{\prime}$ многообразия Блоха-Флоке не влияет на обычный спектр в гильбертовом пространстве функций на всей плоскости $\mathbb{R}^{2}$. Можем ли мы использовать это для решения физически осмысленных (т. е. самосопряженных) краевых задач?

Напомним следующее: для одномерного самосопряженного периодического оператора Шрёдингера $L=-\partial_{x}^{2}+u, u(x+T)=u(x)$, двулистная риманова поверхность Г функций Блоха-Флоке $L \psi=\lambda \psi, T^{*} \psi=\psi(x+T)=\varkappa \psi$ такова, что все ее точки ветвления $a_{s}, s \geqslant 0$, вещественны. Точки ветвления являются границами раздела между спектральными зонами и запрещенными зонами на вещественной прямой. Для того чтобы восстановить потенциал, нам также необходимо знать положения полюсов функции $\psi\left(\lambda, x, x_{0}\right), \lambda \in \Gamma$ (нормализованной условием $\left.\psi\left(x_{0}\right)=1\right)$. Полюсы $\gamma_{j} \in \Gamma$ образуют "дивизор $D$ ". Внутри каждой конечной зоны для гладких потенциалов существует в точности один полюс:

$$
\gamma_{j}\left(x_{0}\right) \in\left[a_{2 j-1}, a_{2 j}\right] .
$$

Нули функции $\psi$ расположены в точках $\gamma_{j}(x)$. Следовательно, наша функция Блоха-Флоке $f\left(x^{*}\right)=\psi\left(\gamma_{j}(x), x^{*}\right)$ удовлетворяет граничной задаче Дирихле на интервале $x^{*} \in[x, x+T]$, где $T$ является периодом, так как $f(x)=f(x+T)=0$.

Другая интерпретация следует из того факта, что та же функция $f\left(x^{*}\right)$ возрастает слева или справа от нуля $\left.f\right|_{x^{*}=x}=0$ и отвечает листу римановой поверхности, где лежит полюс $\gamma\left(x_{0}\right)$. Таким образом, функция $f\left(x^{*}\right)$ принадлежит дискретному спектру на соответствующей полупрямой $x^{*} \in[x,+\infty]$ или $x^{*} \in[-\infty, x]$. 
Вопрос: можем ли мы найти похожую реализацию для блоховских функций $\psi^{\prime}, \psi^{\prime \prime}$ в случае периодического гладкого топологически тривиального магнитного поля $B=(1 / 2) \Delta \ln c$, где $c$ является вещественным и ненулевым?

Классическая теория Дж. фон Неймана самосопряженных граничных условий (как указал И. М. Гельфанд С. П. Новикову в начале 1970-х годов) следует схеме, которая достаточно похожа на тогдашние идеи Новикова построить "эрмитову K-теорию" над кольцами с инволюцией: самосопряженные расширения симметрических операторов отвечают лагранжевым подпространствам в гамильтоновом модуле над кольцом функций. Пусть $\gamma \subset T^{2}$ является контуром в торе, представляющем границу нашей области (или ее связной компоненты).

9.1. Граничные условия для операторов Паули без смешивания компонент. Рассмотрим сначала "скалярные" граничные условия для операторов $L=L^{+}=Q Q^{+}$или $L=L^{-}=Q^{+} Q$ по отдельности, не перемешивая различные компоненты. Мы сводим интеграл к интегралу по границе:

$$
\iint_{D}[(L \psi) \bar{\phi}-\psi(\overline{L \phi})] d^{2} x=\oint_{\gamma}\left[\psi_{1} \bar{\phi}_{2}-\psi_{2} \bar{\phi}_{1}\right] d t
$$

где $\psi_{1}(t)=\left.\nabla_{n} \psi\right|_{\gamma}=\left.\left(\partial_{n}+A_{n}\right) \psi\right|_{\gamma}$ и $\psi_{2}(t)=\left.\psi\right|_{\gamma}$. Здесь $n-$ вектор внешней нормали к границе. Сделать самосопряженное расширение означает выбрать “лагранжево подпространство" $\left(\psi_{1}(t), \psi_{2}(t)\right) \in \Lambda$ для косоэрмитовой граничной формы такое, что

$$
\oint_{\gamma}\left[\psi_{1} \bar{\psi}_{2}^{\prime}-\psi_{2} \bar{\psi}_{1}^{\prime}\right] d t=0
$$

для каждой пары элементов в подпространстве $\Lambda$. Рассмотрим различные типы граничных условий.

"Ультралокальные" граничные условия, не содержащие производных вдоль касательных направлений к границе. Такие граничные условия эллиптичны. K ним относятся:

1) граничные условия Дирихле $\psi_{2}=\left.\psi\right|_{\gamma}=0$;

2) граничные условия Неймана $\psi_{1}=\left.\nabla_{n} \psi\right|_{\gamma}=0$;

$3)$ граничные условия Леонтовича $\alpha(t) \psi_{1}+\beta(t) \psi_{2}=0$ с вещественным ненулевым для всех $t \in \gamma$ вектором $(\alpha, \beta)$; инвариант здесь - отношение $(\alpha(t) / \beta(t))^{ \pm 1}$; это граничное условие имеет топологический заряд (степень отображения $\gamma=$ $\left.S^{1} \rightarrow S^{1}=\mathbb{R} P^{1}\right)$.

Общие “локальные" граничные условия. Такие граничные условия не эллиптичны.

Пусть дана пара скалярных дифференциальных операторов $U, V$ на окружности, один из которых обратим (например, равен 1). Нашим граничным условием является $U \psi_{1}=V \psi_{2}$. Это граничное условие является самосопряженным, если $\left(U V^{+}\right)^{+}=U V^{+}=V U^{+}$. В специальном случае один из операторов равен единице, а другой - произвольному самосопряженному оператору на границе.

Интересен специальный случай, когда $U=1$ и $V$ - оператор первого порядка: $V=i \alpha \partial_{t}+v(t), \alpha, v(t) \in \mathbb{R}$. Граничное условие вида $\psi_{1}=\nabla_{n} \psi=$ $\pm i \psi_{t}+v(t) \psi$ мы назовем $\partial$-bar-граничным условием или $\partial$-граничным 
условием. Причина для этого следующая: мы можем записать это граничное условие (для знака -) в виде

$$
\left[Q^{+} \psi-e^{i \theta(t)} v(t) \psi\right]_{\gamma}=0
$$

где $Q^{+}=-\left(\partial_{\bar{z}}-A_{\bar{z}}\right)$ и $A_{1}=i \Phi_{y}, A_{2}=-i \Phi_{x}, A_{\bar{z}}=-\Phi_{\bar{z}}$, функция $\theta(t)$ является угловым вращением, переводящим координатный репер $x, y$ в репер $\Phi$ рене $n, \partial_{t}$. Граничное условие $\partial$-bar является самосопряженным, но не ультралокальным. Оно не является эллиптическим. Мы не можем найти каких-либо следов этого граничного условия в литературе, хотя оно очень близко к эллиптическому граничному условию и самосопряжено. Можно представить, что такое граничное условие появится в задачах физики. Заменяя опеpaтор $V$ на $V^{\prime}=+i \partial_{t}+v(t)$, мы получим похожее "Ә-граничное условие", где $Q^{+}$заменен на $Q=\partial-\Phi_{z}$. Оно имеет похожие свойства.

Существуют также общие нелокальные самосопряженные граничные условия, однако мы здесь нелокальные граничные условия не рассматриваем.

9.2. Граничные условия для операторов Паули со смешиванием компонент. Оператор $L^{P}$ действует на вектор-функции $\Psi=\left(\psi^{+}, \psi^{-}\right)$. После вычислений мы получим

$$
\left\langle L^{P} \Psi, \Phi\right\rangle-\left\langle\Psi, L^{P} \Phi\right\rangle=\oint_{\gamma}\left\{\left[\psi_{1}^{+} \bar{\phi}_{2}^{+}-\psi_{2}^{+} \bar{\phi}_{1}^{+}\right]+\left[\psi_{1}^{-} \bar{\phi}_{2}^{-}-\psi_{2}^{-} \bar{\phi}_{1}^{-}\right]\right\} d t,
$$

где

$$
\psi_{1}^{ \pm}=\left.\nabla_{n} \psi^{ \pm}\right|_{\gamma}, \quad \psi_{2}^{ \pm}=\left.\psi^{ \pm}\right|_{\gamma} .
$$

Ультралокальные граничные условия. Эти условия эллиптичны.

Наша косоэрмитова форма имеет сигнатуру $(2,2)$ в каждой точке границы $t \in \gamma$. Семейство всех лагранжевых подпространств изоморфно группе $U_{2}$ для всех $t \in \gamma$. Таким образом, все семейство ультралокальных самосопряженных граничных условий может быть классифицировано гладкими отображениями $S^{1} \rightarrow U_{2}$ с топологическим зарядом из группы $\pi_{1}\left(U_{2}\right)=\mathbb{Z}$. Лагранжевы подпространства в каждой точке $t \in \gamma$ могут иметь следующие три типа.

1. Подпространства вида

$$
\psi_{1}^{\alpha}=\sum_{\beta} R^{\alpha, \beta} \psi_{2}^{\beta}
$$

где матрица $R$ эрмитова и $\alpha, \beta= \pm$.

2. Аналогичные подпространства с переставленными числами 1 и 2 : $\psi_{2}=R \psi_{1}$.

Пересечением типов 1 и 2 является в точности подкласс, для которого эрмитова матрица $R$ невырождена. Типы 1 и 2 могут быть объединены формой записи $U \psi_{1}=V \psi_{2}$, где одна из матриц $U, V$ обратима, а матрица $U V^{+}=V U^{+}$ эрмитова.

3. Подпространства вида $a \psi_{1}^{+}+b \psi_{1}^{-}=0, c \psi_{2}^{+}+d \psi_{2}^{-}=0$. 
Свойство лагранжевости влечет $a \bar{c}+b \bar{d}=0$. Мы можем нормализовать эти уравнения, сведя их к виду $|a|^{2}+|b|^{2}=1,|c|^{2}+|d|^{2}=1$. Мы приходим к произвольной матрице из группы $\mathrm{SU}_{2}$, которая нумерует такие специальные лагранжевы подпространства (или произвольные единичные векторы $(a, b) \in \mathbb{C}^{2}$, определяющие всю матрицу).

Общее локальное граничное условие, перемешивающее компоненты. Мы можем написать естественный дифференциальный аналог описанных выше скалярных типов в виде

$$
U \psi_{1}=V \psi_{2}, \quad \psi_{1}=\nabla_{n} \psi, \quad \psi_{2}=\psi
$$

где один из двух операторов $U, V$ на окружности является обратимым. Нашим требованием является $U V^{+}=V U^{+}$.

Наиболее интересными для нас являются граничные условия, содержащие производные обеих компонент $\Psi$ в граничных точках:

$$
\begin{aligned}
\nabla_{n} \psi^{+} & =A^{+} \psi^{+}+b \nabla_{n} \psi^{-}, \\
-\psi^{-} & =c \psi^{+}+d \nabla \psi^{-},
\end{aligned}
$$

где $A^{+}=\alpha^{+} i \partial_{\tau}+a$ есть оператор первого порядка вдоль границы. Здесь все функции $\alpha^{+}(t), a(t), d(t) \in \mathbb{R}$ и $b(t), c(t)$ являются гладкими и $c=\bar{b}$.

Это граничное условие не эллиптично, если порядок оператора $A^{+}$выше нуля. Ультралокальный случай отвечает случаю нулевого порядка $\alpha^{+}=0$.

\section{0. Блоховские функции вне спектра как решения краевых задач}

Обсудим следующую задачу. Пусть $\Psi=\left(\psi^{+}, \psi^{-}\right)$- нулевая мода оператора Паули: $L^{P} \Psi=0$. Для каких контуров это решение удовлетворяет самосопряженным граничным условиям?

Мы рассмотрим эту задачу в обоих случаях: со смешиванием и без смешивания компонент.

Нашей целью является показать, что для некоторых специальных граничных условий эти контуры могут быть описаны как траектории некоторой динамической системы (слоения) на двумерном торе $T^{2}$ или на накрывающем пространстве $\mathbb{R}^{2}$. Сначала рассмотрим ультралокальный случай.

Tеорема 4. Пусть $d=0 u \alpha^{+}=0$. Коэффициенты $a, c, b$ удовлетворяют условиям $a \in \mathbb{R}$ и $c=\bar{b}$ тогда и только тогда, когда граничная кривая $\gamma=\partial D$ является слоем слоения $\omega=0$, где

$$
\omega=\left(\left|\psi^{+}\right|^{2}+\left|\psi^{-}\right|^{2}\right) d \Phi+\left|\psi^{+}\right|^{2} * d \theta^{+}+\left|\psi^{-}\right|^{2} * d \theta^{-} .
$$

Здесъ $\psi^{ \pm}=\left|\psi^{ \pm}\right| \exp \left\{i \theta^{ \pm}\right\} \quad u * d \Phi$ является вектор-потенииалом магнитного поля, звезда * является стандартным оператором двойственности 1-форм на комплексной плоскости.

Случай $b=0$ ведет к граничным условиям без смешивания. Мы детально обсудим этот случай ниже. Для $\partial$-bar-задачи имеем $\alpha^{+}=-1$. Предположим, как и выше, что $d=0$, тогда мы приходим к аналогичной динамической системе. Она детально исследована ниже в случае без смешивания $b=c=0$. 
Доказательство этой теоремы не очень сложное. Оно может быть получено элементарными рассуждениями.

Наиболее интересным для нас является случай $\psi^{+}=\psi^{\prime}, \psi^{-}=\lambda \psi^{\prime \prime}$, где $\lambda$ - параметр и $\psi^{\prime \prime}=Q^{+} \psi^{\prime}$. Здесь $Q^{+}$является оператором, который в квантовой теории трактуется как суперсимметрия для $L^{P}$ (см. раздел 3 ). Наша динамическая система зависит от $\lambda$. При $\lambda=0$ мы имеем случай без смешивания.

Эта пара определяет общее блоховское решение на нулевом уровне энергии для физического оператора Паули с некоторыми (неунитарными) мультипликаторами, так же как было в одномерном случае, где мы имели блоховские решения в запрещенных зонах в качестве собственных функций самосопряженной граничной задачи.

Сделаем некоторые общие замечания, касающиеся граничных задач без смешивания.

Пусть контур $\gamma \subset T^{2}$ задан для следующей области: $D=T^{2} \backslash \gamma$. Найдем решение граничной задачи такой, что $L^{+} \psi=0$ в $D$, с ультралокальными граничными условиями. Пусть

$$
\alpha(t) \nabla_{n} \psi+\beta(t) \psi=0
$$

на контуре $\gamma$. Рассмотрим сначала задачу с контуром, гомотопным нулю в $T^{2}$ (см. рис. $\left.4 \mathrm{a}\right)$, и $\partial D=\gamma$. Решение $\psi$ граничной задачи "типа Леонтовича", удовлетворяющее соотношению (6), мы будем строить, используя интегральное уравнение

$$
\psi=\iint_{\mathbb{R}^{2}}\left[p(k) \psi^{\prime}+q(k) \psi^{\prime \prime}\right] d^{2} k .
$$

Для задачи с контурами $\gamma$, не гомотопными нулю в $T^{2}$, мы имеем $\partial D=\gamma \cup \gamma^{\prime}$ (см. рис. 4c). Существует естественное $\mathbb{Z}$-накрытие $\widehat{D} \rightarrow D$, где $\widehat{D}$ является полосой в $\mathbb{R}^{2}$ со средним направлением $g=[\gamma] \in H_{1}\left(T^{2}, \mathbb{Z}\right)=\mathbb{Z}^{2}$ (см. рис. 4b). Элемент $g \in \mathbb{Z}^{2} \subset \mathbb{C}=\mathbb{R}^{2}$ действует свободно, $g: \widehat{D} \rightarrow \widehat{D}$, как сдвиг на комплексное число:

$$
g: \quad z \rightarrow z+g, \quad \bar{z} \rightarrow \bar{z}+\bar{g} .
$$

По определению, компоненты границы

$$
\partial \widehat{D}=\widehat{\gamma} \cup \widehat{\gamma}^{\prime}
$$

являются накрытиями над $\gamma, \gamma^{\prime}$. Элемент $g$ можно рассматривать как вектор решетки $\mathbb{Z}^{2}$ в $\mathbb{R}^{2}$. Другой порождающий элемент $g^{\prime} \in \mathbb{Z}^{2}$, дополнительный к $g$, отображает в точности контур $\gamma$ в контур $\gamma^{\prime}, g^{\prime}: \gamma \rightarrow \gamma^{\prime}$. Мы будем говорить, что $\gamma^{\prime}\left(\widehat{\gamma}^{\prime}\right)$ находится справа от $\gamma(\widehat{\gamma})$, если $\left|\varkappa\left(g^{\prime}\right)\right|<1$. Пусть $c \neq 0$. Все функции $\psi^{\prime}(k), \psi^{\prime \prime}(k)$ с $k$, ортогональными $g$, имеют унитарные блоховские мультипликаторы $|\varkappa(g)|=1$ вдоль сдвига $g$. Мы имеем четыре области: $D_{+}, D_{-}, \widehat{D}$ в $\mathbb{R}^{2}$ и $D$ в $T^{2}$ (см. рис. $\left.4 \mathrm{a}-4 \mathrm{~d}\right)$, при этом

$$
D_{+} \cup D_{-}=\mathbb{R}^{2}, \quad D_{+} \cap D_{-}=\widehat{\gamma}, \quad g^{\prime}\left(D_{-}\right) \subset D_{-}, \quad g^{\prime}\left(D_{+}\right) \cap D_{-}=\widehat{D} .
$$

Мы требуем, чтобы на контурах $\gamma, \gamma^{\prime}$ выполнялось равенство

$$
\alpha(t) \nabla_{n} \psi+\beta(t) \psi=0,
$$


но допускаем различные пары коэффициентов $(\alpha, \beta),\left(\alpha^{\prime}, \beta^{\prime}\right)$ для $\gamma$ и $\gamma^{\prime}$ в области $\widehat{D}$ такой, что оба контура входят в границу. Функция $\psi$ должна быть построена существенным образом по $\psi^{\prime}$, но, если необходимо, мы можем добавить $\psi^{\prime \prime}$. Наиболее общая возможность является такой, что $\psi$ задана интегралом вдоль $k$-оси, ортогональной $g$ :

$$
(\mathrm{I}): \quad \psi=\int_{k \geqslant 0}\left[\psi^{\prime}(k, x, y) p(k)+\psi^{\prime \prime}(k, x, y) q(k)\right] d k
$$

для полуплоскости $D_{+} \subset \mathbb{R}^{2}$;

$$
\text { (II) }: \quad \psi=\int_{k \leqslant 0}\left[\psi^{\prime}(k, x, y) p(k)+\psi^{\prime \prime}(k, x, y) q(k)\right] d k
$$

для полуплоскости $D_{-} \subset \mathbb{R}^{2}$;

$$
\text { (III) : } \quad \psi=\int_{k \in \mathbb{R}}\left[\psi^{\prime}(k, x, y) p(k)+\psi^{\prime \prime}(k, x, y) q(k)\right] d k
$$

для полосы $\widehat{D} \subset \mathbb{R}^{2}$;

$$
(\mathrm{IV})_{\varkappa}: \quad \psi_{\varkappa}=\sum_{m \in \mathbb{Z}}\left[\psi^{\prime}\left(k_{m}, x, y\right) p_{m}(\kappa)+\psi^{\prime \prime}\left(k_{m}, x, y\right)\right] q_{m}(\kappa)
$$

для $D \subset T^{2}$, где соответствующее $k_{m}$ таково, что все $\psi^{\prime}\left(k_{m}\right), \psi^{\prime \prime}\left(k_{m}\right)$ имеют один и тот же самый фиксированный унитарный мультипликатор $\varkappa=e^{k_{m} \bar{g}}$ (т. е. $k_{m}=k_{0}+2 \pi i m / \bar{g}$, где $m \in \mathbb{Z}$ ).

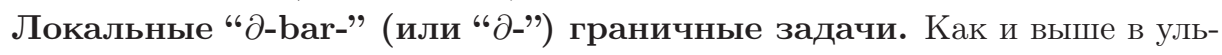
тралокальном случае, мы имеем два типа контуров:

- задача 1 с контурами $\gamma$, гомотопными нулю в $T^{2}$, где $\gamma=\partial D$;

- задача 2 с контурами $\gamma \subset T^{2}$, не гомотопными нулю на $T^{2}$; здесь $\partial D=\gamma \cup \gamma^{\prime}$.

Во втором случае (наиболее интересном для нас) мы рассматриваем те же типы областей $D_{+}, D_{-}, \widehat{D}, D$, что и выше, с границами, не гомотопными нулю в $T^{2}$, и их накрывающими в $\mathbb{R}^{2}$ (см. рис. $\left.4 \mathrm{a}-4 \mathrm{~d}\right)$.

Рис. $4 \mathrm{a}$ и $4 \mathrm{~b}$ отвечают примеру 2 (см. ниже). Здесь $g$ параллелен среднему направлению $\widehat{\gamma}$.

Рис. 4c и $4 \mathrm{~d}$ отвечают примеру 1. Контур $\widehat{\gamma}$ является просто прямой $x=0$, $-\infty<y<\infty$ (см. рис. 4c).

Наше граничное соотношение вдоль границы (возможно, с различными коэффициентами для различных компонент границы) имеет вид

$$
Q^{ \pm} \psi=v(t) \psi e^{i \theta(t)}
$$

или

$$
\nabla_{n} \psi=\left( \pm i \partial_{t}+u(t)\right) \psi
$$

для вещественных $u, v$. Мы можем пытаться найти решение $\psi$, используя, как и выше, интегральные уравнения. 


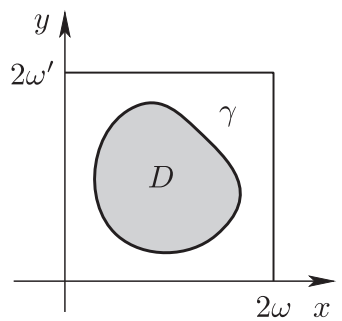

$D \subset T^{2}$

Рис. 4а. Контур $\gamma$ гомотопен нулю

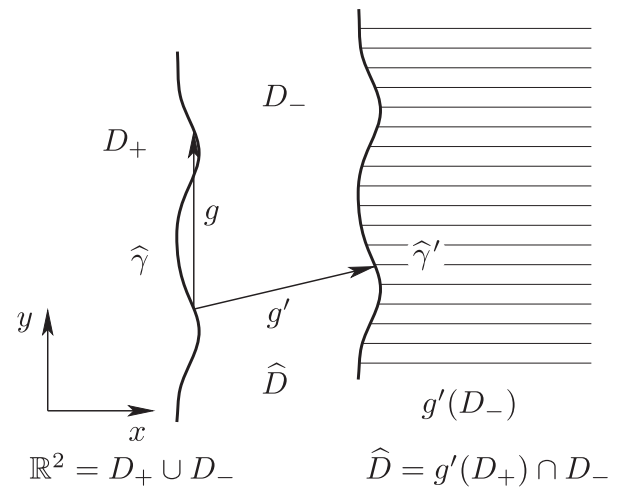

Рис. 4b. Контур $\gamma$ не гомотопен нулю
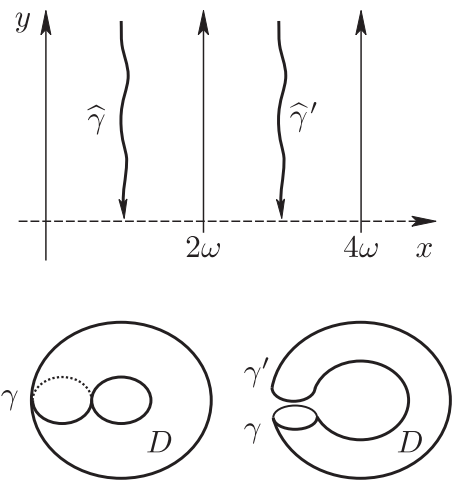

$D=T^{2} \backslash \gamma, \partial D=\gamma \cup \gamma^{\prime}$

Рис. 4c

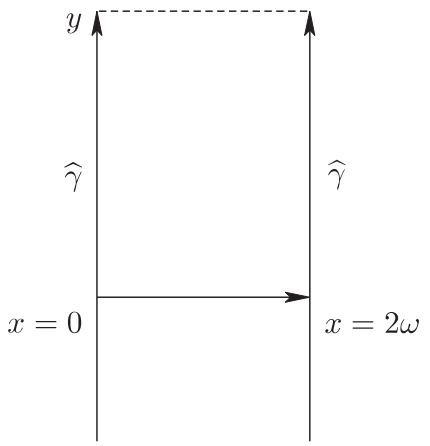

Рис. $4 \mathrm{~d}$

Мы уже обсуждали выше следующий вопрос: какие общие ограничения должны быть выполнены для класса функций $\psi$, решающих задачу типа Леонтовича в магнитном поле с вектор-потенциалом

$$
A=-i * d \Phi=i\left(-\Phi_{y} d x+\Phi_{x} d y\right) ?
$$

Теперь мы будем рассматривать только случай без смешивания. Предполагая, что $\psi=\rho(x, y) e^{i \theta(x, y)}$, можно видеть, что отношение $\nabla_{n} \psi / \psi$ должно быть вещественным. Здесь $n$ - нормаль к границе $\gamma \subset \partial D$. Мы приходим к следующему утверждению (частный случай сформулированной выше теоремы 4).

Лемма 3. Если фазовая функиия $\theta(x, y)$ известна, то граничный контур $\gamma$ для самосопряженного скалярного оператора $L \psi=\lambda \psi$ с любым граничным условием типа Леонтовича должен быть слоем слоения, заданным уравнением $*(d \theta+A / i)=\Omega=0$ или $\left(\theta_{y}+\Phi_{x}\right) d x+\left(-\theta_{x}+\Phi_{y}\right) d y=0$. И наоборот, если контур $\gamma$ известен, то вдоль контура должно быть выполнено ограничение $* d \theta=-d \Phi$. 


\section{Специальные контуры, для которых решение несмешанной крае-} вой задачи является блоховской функцией $\psi^{\prime}$.

Пример 1. $\psi^{\prime}$ как решение граничной задачи Леонтовича для специальных контуров первого типа. Пусть $g=0$. Мы строим решение с $2 n+1$ точками пересечения $p_{j}, k_{j}$, где

$$
p_{j}=a+i b_{j}, \quad p_{n+j}=-p_{j}, \quad k_{n+j}=-k_{j} .
$$

Пусть задан набор констант (не имеющих ничего общего с блоховскими мультипликаторами) $\kappa_{n+j}=\kappa_{j}$ и все параметры $a, b_{j}, \kappa_{j}$ вещественны. Пусть $\sum_{j=1}^{n} \kappa_{j}=0$. Имеем

$$
\begin{gathered}
c=\kappa_{0}+2 \sum_{j=1}^{n} \kappa_{j} \cos \left(2\left(b_{j} x+a y\right)\right) \\
\frac{\psi^{\prime}}{k}=e^{k \bar{z}}\left(\frac{\kappa_{0}}{k}+\sum_{j=1}^{n}\left(\kappa_{j} \frac{e^{p_{j} z-k_{j} \bar{z}}}{k-k_{j}}+\kappa_{n+j} \frac{e^{p_{n+j} z-k_{n+j} \bar{z}}}{k-k_{n+j}}\right)\right) .
\end{gathered}
$$

Для $x=0$ имеем $c=\kappa_{0}=$ const. Мы назовем такой контур специальным контуром первого типа. Возьмем теперь контур $\gamma$, заданный равенством $x=0$ или $c=$ const, и рассмотрим граничную задачу

$$
\psi_{n}=\alpha(x) \psi .
$$

Укажем здесь, что для таких контуров выполнено соотношение $\nabla_{n}=\partial_{n}$, так как $d \Phi=0$ на контуре $\Phi=\ln c=$ const и $A=\left(i \Phi_{y},-i \Phi_{x}\right)$. Должно быть выполнено условие $\psi_{x}^{\prime} / \psi \in \mathbb{R}$ при $x=0$. Представляя $\psi^{\prime}$ в виде $\psi^{\prime}=e^{k \bar{z}}(f+i g)$, мы видим, что условие $\psi_{x}^{\prime} /\left.\psi^{\prime}\right|_{x=0} \in \mathbb{R}$ следует для $k \in \mathbb{R}$ из требований $\left.g\right|_{x=0}=$ $\left.g_{x}\right|_{x=0}=0$. После элементарных вычислений мы видим, что

$$
\left.g\right|_{x=0}=A_{1} \cos (a y)+A_{2} \sin (a y) \quad \text { и }\left.\quad g_{x}\right|_{x=0}=B_{1} \cos (a y)+B_{2} \sin (a y),
$$

где константы $A_{q}, B_{q}$ линейно выражаются через константы $\kappa_{j}$. Наше граничное условие эквивалентно четырем однородным линейным уравнениям $A_{1}=$ $A_{2}=B_{1}=B_{2}=0$. Таким образом, при $n>5$ мы можем найти решение для каждого фиксированного значения констант $a, b_{j}, k \in \mathbb{R}$. Вещественные значения $k$ ортогональны направлению контура $x=0$. Итак, мы доказали следующую лемму.

Лемма 4. Для каждого множества экспонент с нечетным числом $2 n+1$ точек самопересечения, где $n>5$, существует дивизор (т.е. множество констант $\left.\kappa_{j}\right)$ такой, что соответствующая функиия Блоха-Флоке $\psi^{\prime}(k, x, y)$ c вещественным $k \in \mathbb{R}$ удовлетворяет граничному условию Леонтовича $\psi_{x}^{\prime} / \psi^{\prime} \in \mathbb{R}$ для контура $\gamma: x=0$. Блоховский мультипликатор вдоль направления контура является унимодулярным: $\kappa=e^{-i k y}$.

Таким образом, наша задача является самосопряженной. Соответствующая функция $\psi^{\prime}(k, x, y)$ служит решением граничной задачи Леонтовича в области $\widehat{D}: 0<x<2 \omega$ и в одной из двух полуплоскостей $D_{+}: 0<x$ или $D_{-}: x<0$ в зависимости от знака соответствующего значения $k$. Она также обслуживает спектр в области $D$, где $x \in[0,2 \omega]$, в торе $T^{2}$ (т. е. периодична по переменной $y$ ), см. рис. 4 с и $4 \mathrm{~d}$. 
Рис. 5 отвечает следующему ниже примеру 2 , посвященному “д-bar"- граничным условиям. В этом примере мы имеем “максимальный” граничный контур, гомотопный нулю, такой, что площадь внутри совпадает со всей площадью тора. В частности, магнитный поток через эту область равен нулю согласно нашим предположениям на класс магнитных полей, которые мы рассматриваем. В этом случае граничный цикл состоит из сепаратрис (см. рис. 5).

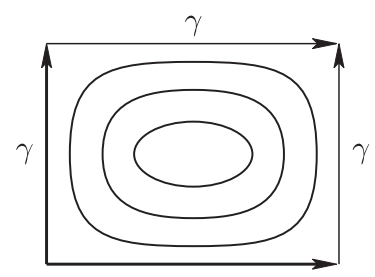

$\gamma$ $K$

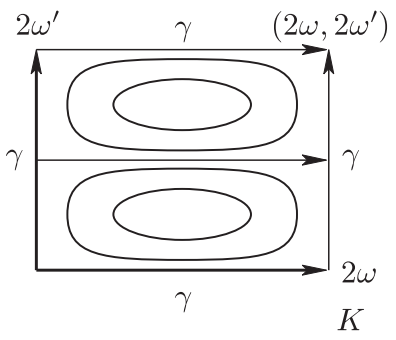

b)

Рис. 5

Пример 2. $\psi^{\prime}$ как решение "д-bar"-граничной задачи для специального контура второго вида. Мы пишем "д-bar"-задачу в виде

$$
Q^{+} \psi=u(t) e^{i \theta(t)} \psi
$$

где $u \in \mathbb{R}$ и $\theta(t)$ - угол между репером $x, y$ и репером Френе $n, \partial_{\tau}$ вдоль граничной кривой $\gamma$. Пусть $\psi=\psi^{\prime}(k, x, y) / \sqrt{c}$. Имеем $Q^{+} \psi / \psi=e^{i \theta_{k}(x, y)} u_{k}(x, y)$, где $u_{k} \in \mathbb{R}$. Таким образом, наш контур $\gamma$ должен быть касательным к векторному (директорному) полю $\partial_{\tau}$ на торе. Это поле зависит от параметра $k \in \mathbb{C}$. Оно получено из направления $\partial_{x}$ вращением $e^{i \theta_{k}(x, y)}$ в точке $x, y$ или $z=x+i y$. Это векторное (директорное) поле может быть описано нулями 1-формы:

$$
\operatorname{Re}\left[\frac{Q^{+} \psi}{\psi} d \bar{z}\right]=0 .
$$

Пусть

$$
c=1+\sum_{j=1}^{n}\left(a_{j} e^{l_{j} \bar{z}-\bar{l}_{j} z}+\bar{a}_{j} e^{-l_{j} \bar{z}+\bar{l}_{j} z}\right),
$$

и в новой нормировке, где полюсы расположены в точках пересечения, имеем

$$
\frac{\psi^{\prime}}{k}=\left(\frac{1}{k}+\sum_{j=1}^{n}\left(a_{j} \frac{e^{l_{j} \bar{z}-\bar{l}_{j} z}}{k+l_{j}}+\bar{a}_{j} \frac{e^{-l_{j} \bar{z}+\bar{l}_{j} z}}{k-l_{j}}\right)\right) e^{k \bar{z}} .
$$

Теперь сделаем калибровочное преобразование $\psi=\psi^{\prime} / \sqrt{c}$, приводящее оператор к самосопряженному виду $L=Q Q^{+}$. Имеем $Q^{+} \psi / \psi=-\bar{\partial} \psi^{\prime} / \psi^{\prime}$. Но $\bar{\partial}\left(\psi^{\prime} / k\right)=2 c e^{k \bar{z}}$ для $\psi^{\prime}$ с новой нормировкой. В результате получим

$$
\frac{Q^{+} \psi}{\psi}=-\frac{2 c k e^{k \bar{z}}}{\psi^{\prime}}
$$


Следовательно, мы описали множество контуров, для которых $\partial$-bar-граничное условие имеет в качестве решения функцию $\psi^{\prime}(k, x, y)$. Мы назовем их специальными контурами второго рода. Эти контуры являются слоями слоения на двумерном торе, заданном следующим уравнением:

$$
\frac{c k e^{k \bar{z}}}{\psi} d \bar{z}+\frac{c \bar{k} e^{\bar{k} z}}{\bar{\psi}} d z=0
$$

на $\gamma$. Здесь $c$ вещественно и отлично от нуля.

Нули $\psi^{\prime}$ являются сингулярными точками этого слоения. Многообразие нулей $N_{0}=\left\{\psi^{\prime}(k, x, y)=0\right\}$ является компактным и двумерным, кроме того, $N_{0} \subset \mathbb{C} \times \mathbb{R}^{2}=\{k, x, y\}$. Пересечение $N_{0}$ с каждой плоскостью $k=$ const coстоит из конечного числа изолированных точек. Поверхность $N_{0}$ проектируется в $k$-плоскость. Образ является компактным множеством и $N_{0} \rightarrow N^{*} \subset \mathbb{C}$. Мы знаем, что $N^{*}$ является компактным и не покрывает 0 . Для всех $k \in \mathbb{C} \backslash N^{*}$ наше слоение на двумерном торе не имеет сингулярных точек. Наш поток является аналитическим. Согласно известным классическим результатам, такой поток всегда гомеоморфен прямолинейному потоку с некоторым "числом вращения" $\rho(k)$. Таким образом, мы имеем

$$
\frac{\overline{\psi^{\prime}}}{\bar{k} e^{k \bar{z}}} d \bar{z}+\frac{\psi^{\prime}}{k e^{k \bar{z}}} d z=0 .
$$

Введем вещественную функцию

$$
F=\left(\frac{1}{|k|^{2}}+\sum_{j}^{n}\left(a_{j} \frac{e^{l_{j} \bar{z}-\bar{l}_{j} z}}{\left(k+l_{j}\right)\left(\bar{k}-\bar{l}_{j}\right)}+\bar{a}_{j} \frac{e^{-l_{j} \bar{z}+\bar{l}_{j} z}}{\left(k-l_{j}\right)\left(\bar{k}+\bar{l}_{j} z\right)}\right)\right) e^{k \bar{z}+\bar{k} z} .
$$

Легко проверить, что $\partial F=2 \psi^{\prime} /\left(k e^{\bar{k} z}\right)$ и $\bar{\partial} F=2 \overline{\psi^{\prime}} /\left(\bar{k} e^{k \bar{z}}\right)$. Для всех $k \in \mathbb{C} \backslash N^{*}$ контуры глобально заданы уравнением $d F=0$ или $F=$ const в $\mathbb{R}^{2}$. Для всех значений $k \in \mathbb{C}$ несингулярные уровни $F=a$ определяют траектории, которые не достигают сингулярных точек. Все эти траектории либо компактны и гомотопны нулю, либо топологически эквивалентны в $\mathbb{R}^{2}$ прямолинейным траекториям вокруг тора с некоторым числом вращения. Все компоненты уровня $F=$ const, содержащие сингулярные точки, являются либо изолированными сингулярными точками (центрами слоений), либо сепаратрисами седел (включая вырожденные седла). Мы доказали следующее утверждение.

ЛЕмма 5. Наше слоение эквивалентно гамильтоновой системе (слоению) c гамильтонианом $F$ в каждой компактной области $E$, лежащей в универсальном накрытии $E \subset \mathbb{R}^{2}$.

Как следствие мы получаем, что

(i) все сингулярные точки являются либо центрами, либо седлами (возможно, вырожденными);

(ii) не существует предельных циклов, гомотопных нулю в торе, в том числе предельных циклов, построенных из частей сепаратрис от седел к седлам.

Является ли наше слоение глобально топологически эквивалентным гамильтонову слоению в торе, заданному некоторым многозначным гамильтонианом (или замкнутой 1-формой)? 
Могут ли для нашей системы существовать предельные циклы, не гомотопные нулю? Пусть задан такой цикл $\gamma$. Он может быть несингулярным или состоять из нескольких частей сепаратрис. В любом случае значение функции $F$ вдоль $\gamma$ является константой и число вращения вдоль $\gamma$ является рациональным. Согласно нашему предположению семейство незамкнутых открытых несингулярных траекторий $\gamma_{1}$ приближается к $\gamma$ при $t \rightarrow \infty$. Мы всегда можем выбрать $\gamma_{1}$ так, что $F(\gamma) \neq F\left(\gamma_{1}\right)$.

Существуют следующие два случая.

Случай 1: функиия $\psi^{\prime}$ является неограниченной вдоль замкнутого конту$p a \gamma$. Это возможно только для таких контуров, что $F(\gamma)=0$ (доказательство см. ниже). Любой замкнутый контур, не гомотопный нулю в торе, с неограниченной $\psi^{\prime}$, является пределом для нашего слоения - см. пример к случаю 1 и рис. 6.

ПРИмеР к СЛУЧАЮ 1. Пусть функции $c, F$ такие же, как и выше. Возьмем следующие параметры:

$$
\begin{gathered}
n=2, \quad l_{1}=\frac{1}{2}, \quad l_{2}=\frac{i}{2}, \quad a_{1}=a_{2}=0.2, \quad k=0.55 i, \\
c=1+0.4 \cos x+0.4 \cos y .
\end{gathered}
$$

Решая пример численно, мы приходим к предельным циклам $\gamma$, где $b=[\gamma] \in$ $H_{1}\left(T^{2}, \mathbb{Z}\right)$ и $b$ является базисным циклом вдоль $y$-оси (мнимой). Имеем $F(\gamma)=0$ (см. рис. 6).

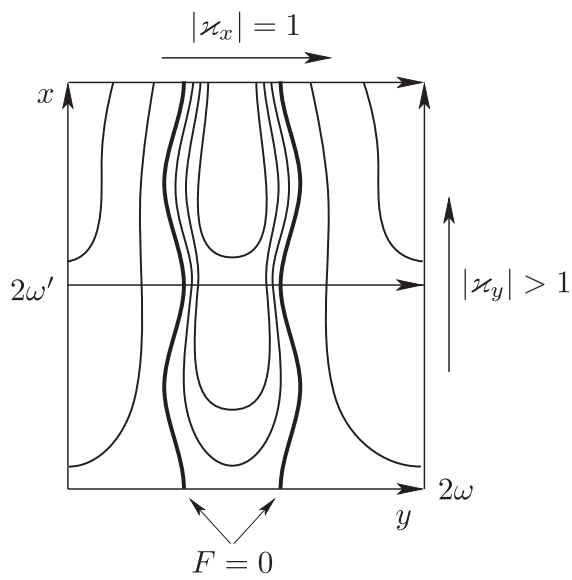

Рис. 6

Здесь контуры $F=0$ замкнуты и не гомотопны нулю. Блоховский мультипликатор в $y$-направлении отличен от 1 . Поэтому дифференциал $d F$ экспоненциально возрастает в $y$-направлении и все контуры $F=c$ стремятся к контуру $F=0$ при $y \rightarrow+\infty$.

Случай 2: Функция $\psi^{\prime}$ ограничена вдоль открытых контуров $\gamma_{1}$. Согласно лемме 6 ниже это всегда верно, если все компоненты связности уровня $F=0$ являются компактными в $\mathbb{R}^{2}$. Число вращения является рациональным для замкнутого контура $\gamma$, не гомотопного нулю. Таким образом, траектория $\gamma$ соответствует сдвигу $g: \mathbb{R}^{2} \rightarrow \mathbb{R}^{2}$ на элемент решетки с унитарным мультипликатором. После $M$ итераций мы получим число вращения 
для $g^{M}$, равное 1 . Но форма $d F$ преобразуется по правилу $g^{*}(d F)=\varkappa d F$ для всех сдвигов $g$ из решетки, причем блоховские мультипликаторы $g^{*} \psi^{\prime}=\varkappa \psi^{\prime}$, $\varkappa \in \mathbb{R}$, вещественны. Мы знаем, что $F=$ const вдоль $\gamma_{1}$ и что форма $d F$ инвариантна при сдвиге $g^{M}$ с унитарным мультипликатором $\varkappa=1$. Итак, мы видим, что $F(\gamma)=F\left(\gamma_{1}\right)$ для всех $\gamma_{1}$, достигающих $\gamma$, если $\gamma$ является несингулярным. Мы получаем противоречие для несингулярных $\gamma$, поскольку $F$ является гладкой (даже аналитической) функцией. Она не постоянна вдоль трансверсального сечения к $\gamma$. Это верно также для сингулярного $\gamma$, построенного по сепаратрисам. Для доказательства этого утверждения мы должны использовать вместо $d F$ специфику блоховского свойства функции $F$, которая задана явными формулами. В частности, мы должны воспользоваться ее поведением при сдвигах на элементы решетки вместо рассмотрения формы $d F$.

Таким образом, мы заключаем, что наше слоение не может иметь несингулярных (и сингулярных) предельных циклов, гомотопных нулю и таких, что $F(\gamma) \neq 0$.

Лемма 6. Пусть $\widehat{\gamma}$ является открытой несингулярной траекторией такой, что топологическое замыкание ее проекиии $\gamma$ в торе $T^{2}$ не содержит уровней $F=0$. Тогда $\psi^{\prime}$ ограничена вдоль контуров $\gamma$. Для каждого несингулярного периодического поля $c \neq 0$ и достаточно большого $|k|$ это условие выполнено.

Доказательство немедленно следует из формул для функций $F$ и $\psi^{\prime}$ при достаточно большом $|k|$. Функцию $\psi^{\prime}$ можно представить в виде произведения $e^{k \bar{z}}$ и ограниченной периодической функции. Таким образом, $\left|\psi^{\prime}\right| \sim e^{\operatorname{Re}(k \bar{z})}$. Функция $F$ представима в виде периодической функции, умноженной на функцию $e^{2 \operatorname{Re}(k \bar{z})}$. Таким образом, функция $e^{\operatorname{Re}(k \bar{z})}$ ограничена вдоль уровней $F=$ const $\neq 0$. Поэтому $\psi^{\prime}$ также ограничена вдоль этого контура. Для достаточно большого $|k|$ асимптотика функции $F$ имеет вид $\left(c /|k|^{2}\right) e^{k \bar{z}+\bar{k} z}$, поэтому эта функция отделена от нуля. Лемма доказана.

Мы приходим к выводу, что существуют только следующие три возможности для слоев этого слоения при $k \in \mathbb{C}$.

1. Существует открытая несингулярная траектория $\gamma$, для которой $\psi^{\prime}(k, x, y)$ является решением сомосопряженной $\partial$-bar-граничной задачи в цилиндрической области $D \subset T^{2}$, в ленточной области $\widehat{D}$ и в деформированных полуплоскостях $D_{+}$или $D_{-}$. Необходимо выбрать только одну область $D_{+}$или $D_{-}$, где $\psi^{\prime}(k, x, y) / \sqrt{c}$ убывает на бесконечности в направлении, трансверсальном к границе (см. рис. $4 \mathrm{a}-4 \mathrm{~d})$.

2. Существует "максимальный" цикл $\gamma$, гомотопный нулю на торе $T^{2}$, состоящий из сепаратрис седел такой, что $\gamma$ ограничивает всю площадь тора (см. рис. 5). Функция $\psi^{\prime}$ дает решение самосопряженной граничной задачи.

3. Существует предельный цикл $\gamma$, не гомотопный нулю, такой, что $F(\gamma)=0$ (см. рис. 6). Для такого граничного контура функция $\psi^{\prime}$ не порождает решение самосопряженной задачи, так как она имеет экспоненциальный рост вдоль границы.

Эта картина является естественным 2D-обобщением одномерного случая, где функции $f\left(x^{*}\right)=\psi\left(\gamma_{j}(x), x^{*}\right)$ дают новые уровни в запрещенных зонах спектра периодической задачи и описывают либо дополнительный спектр задачи Дирихле на отрезке $x^{*} \in[x, x+T]$, либо "приграничные" состояния на полупрямой $R^{+}=[-\infty, x]$ или $R^{-}=[x,+\infty]$ для оператора $L=-\partial_{x^{*}}^{2}+u\left(x^{*}\right)$. 


\section{Список литературы}

[1] В. Б. Берестецкий, Е. М. Лифшиц, Л. П. Питаевский, Теоретическая физика, т. 4: Квантовая электродинамика, 2-е изд., Наука, М., 1980, 704 с.; англ. пер.: V.B. Berestetskii, E. M. Lifshitz, L. P. Pitaevskii, Course of theoretical physics, v. 4: Quantum electrodynamics, 2nd ed., Butterworth-Heinemann, 1982, 652 pp.

[2] П. Г. Гриневич, А. Е. Миронов, С.П. Новиков, "Двумерный оператор Шрёдингера: эволюционные $(2+1)$-системы и их новые редукции; двумерная иерархия Бюргерса и данные обратной задач", УМH, 65:3(393) (2010), 195-196; англ. пер.: P. G. Grinevich, A. E. Mironov, S. P. Novikov, "2D-Schrödinger operator, $(2+1)$ evolution systems and new reductions, 2D-Burgers hierarchy and inverse problem data", Russian Math. Surveys, 65:3 (2010), 580-582; 2D Schrodinger operator, $(2+1)$ systems and new reductions. The $2 \mathrm{D}$ Burgers hierarchy and inverse problem data, 2010, 5 pp., arXiv: 1005.0612.

[3] П. Г. Гриневич, А.Е. Миронов, С.П. Новиков, "О нулевом уровне чисто магнитного двумерного нерелятивистского оператора Паули для частиц со спином 1/2", ТМФ, 164:3 (2010), 333-353; англ. пер.: P. G. Grinevich, A.E. Mironov, S. P. Novikov, "Zero level of a purely magnetic two-dimensional nonrelativistic Pauli operator for SPIN-1/2 particles", Theoret. and Math. Phys., 164:3 (2010), 1110-1127; On the ground level of purely magnetic algebro-geometric 2D Pauli operator (spin 1/2), 2010, 32 pp., arXiv: 1004.1157.

[4] П. Г. Гриневич, А. Е. Миронов, С. П. Новиков, “Исправление к статье: 'О нулевом уровне чисто магнитного двумерного нерелятивистского оператора Паули для частиц со спином 1/2' (ТМФ. 2010. Т. 164, № 3. С. 333-353)”, ТМФ, 166:2 (2011), 320; англ. пер.: P. G. Grinevich, A. E. Mironov, S. P. Novikov, "Erratum to: 'Zero level of a purely magnetic two-dimensional nonrelativistic Pauli operator for SPIN-1/2 particles'", Theoret. and Math. Phys., 166:2 (2011), 278.

[5] P. G. Grinevich, A. E. Mironov, S. P. Novikov, On the nonrelativistic 2D purely magnetic supersymmetric Pauli operator, 2011 (v3 - 2015), 39 pp., arXiv: 1101.5678.

[6] П. Г. Гриневич, А. Е. Миронов, С. П. Новиков, “Двумерный оператор Паули в магнитном поле", Физика низких температур, 37:9-10 (2011), 1040-1045; англ. пер.: P. G. Grinevich, A. E. Mironov, S. P. Novikov, "Two-dimensional Pauli operator in a magnetic field", Low Temp. Phys., 37:10 (2011), 829-833.

[7] J.E. Avron, R. Seiler, "Paramagnetism for nonrelativistic electrons and Euclidean massless Dirac particles", Phys. Rev. Lett., 42:15 (1979), 931-934.

[8] Y. Aharonov, A. Casher, "Ground state of a spin- $1 / 2$ charged particle in a twodimensional magnetic field", Phys. Rev. A, 19:6 (1979), 2461-2462.

[9] Б. А. Дубровин, С. П. Новиков, "Основные состояния двумерного электрона в периодическом поле", ЖЭЭТФ, 79:3 (1980), 1006-1016; англ. пер.: В. А. Dubrovin, S.P. Novikov, "Ground states of a two-dimensional electron in a periodic magnetic field", Soviet Phys. JETP, 52:3 (1980), 511-516.

[10] Б. А. Дубровин, С.П. Новиков, "Основные состояния в периодическом поле. Магнитно-блоховские функции и векторные расслоения", Докл. АН CCCP, 253:6 (1980), 1293-1297; англ. пер.: В. A. Dubrovin, S. P. Novikov, "Ground states in a periodic field. Magnetic Bloch functions and vector bundles", Soviet Math. Dokl., 22 (1980), 240-244.

[11] С. П. Новиков, "Магнитно-блоховские функции и векторные расслоения. Типичные законы дисперсии и их квантовые числа", Докл. АН СCCP, 257:3 (1981), 538-543; англ. пер.: S. P. Novikov, "Magnetic Bloch functions and vector bundles. Typical dispersion laws and their quantum numbers", Soviet Math. Dokl., 23:2 (1981), 298-303.

[12] А. С. Лыскова, "Об операторе Шрёдингера в магнитном поле", УМН, 36:2(218) (1981), 189-190; англ. пер.: A.S. Lyskova, "On the Schrödinger operator in a magnetic field", Russian Math. Surveys, 36:2 (1981), 181-182. 
[13] А.С. Лыскова, "Топологические свойства оператора Шрёдингера в магнитном поле и слабом потенциале", УМН, 36:5(221) (1981), 181-182; англ. пер.: A. S. Lyskova, "Topological properties of the Schrödinger operator in a magnetic field and with a weak potential", Russian Math. Surveys, 36:5 (1981), 165-166.

[14] С. П. Новиков, "Двумерные операторы Шрёдингера в периодических полях", Итоги науки и техн. Сер. Соврем. пробл. матем., т. 23, ВИНИТИ, М., 1983, 3-32; англ. пер.: S. P. Novikov, "Two-dimensional Schrödinger operators in periodic fields", J. Soviet Math., 28:1 (1985), 1-20.

[15] D. J. Thouless, M. Kohmoto, M. P. Nightingale, M. den Nijs, "Quantized Hall conductance in a two-dimensional periodic potential", Phys. Rev. Lett., 49:6 (1982), 405-408.

[16] R. Tao, D. J. Thouless, "Fractional quantization of Hall conductance", Phys. Rev. B, 28:2 (1983), 1142-1144.

[17] Л.Э. Генденштейн, "Суперсимметрия в задаче об электроне в неоднородном магнитном поле", Писъма в ЖЭТФ, 39:5 (1984), 234-236; англ. пер.: L. Е. Gendenshtein, "Supersymmetry in the problem of an electron in a nonuniform magnetic field", JETP Lett., 39 (1984), 280-282.

[18] G. Darboux, Leçons sur la théorie générale des surfaces et les applications géométriques du calcul infinitésimal. II, Gauthier-Villars, Paris, 1915, xix+567 pp.

[19] S. P. Novikov, A.P. Veselov, "Exactly solvable 2-dimensional Schrödinger operators and Laplace transformations", Solitons, geometry and topology: on the crossroad, Amer. Math. Soc. Transl. Ser. 2, 179, Amer. Math. Soc., Providence, RI, 1997, 109-132.

[20] Б. А. Дубровин, В.Б. Матвеев, С.П. Новиков, "Нелинейные уравнения типа Кортевега-де Фриза, конечнозонные линейные операторы и абелевы многообразия", УМH, 31:1(187) (1976), 55-136; англ. пер.: B. A. Dubrovin, V. B. Matveev, S.P. Novikov, "Non-linear equations of Korteweg-de Vries type, finite-zone linear operators, and Abelian varieties", Russian Math. Surveys, 31:1 (1976), 59-146.

[21] N. J. Hitchin, "Harmonic maps from a 2-torus to the 3-sphere", J. Differential Geom., 31:3 (1990), 627-710.

[22] А.И. Бобенко, "Поверхности постоянной средней кривизны и интегрируемые уравнения", УМH, 46:4(280) (1991), 3-42; англ. пер.: А. I. Bobenko, "Constant mean curvature surfaces and integrable equations", Russian Math. Surveys, 46:4 (1991), 1-45.

[23] С. В. Манаков, "Метод обратной задачи рассеяния и двумерные эволюционные уравнения", УМН, 31:5(191) (1976), 245-246.

[24] Б. А. Дубровин, И. М. Кричевер, С. П. Новиков, "Уравнение Шрёдингера в периодическом поле и римановы поверхности", Докл. АН СССР, 229:1 (1976), 15-18; англ. пер.: В. A. Dubrovin, I. M. Krichever, S. P. Novikov, "The Schrödinger equation in a periodic field and Riemann surfaces", Soviet Math. Dokl., 17:4 (1976), 947-951.

[25] И. В. Чередник, "Об условиях вещественности в конечнозонном интегрировании", Докл. АН ССCP, 252:5 (1980), 1104-1108; англ. пер.: I. V. Cherednik, "Reality conditions in "finite-zone integration", Soviet Phys. Dokl., 25:6 (1980), 450-452.

[26] А.П. Веселов, С.П. Новиков, "Конечнозонные двумерные потенциальные операторы Шрёдингера. Явные формулы и эволюционные уравнения", Докл. АН CCCP, 279:1 (1984), 20-24; англ. пер.: A. P. Veselov, S. P. Novikov, "Finite-zone, two-dimensional, potential Schrödinger operators. Explicit formulas and evolution equations", Soviet Math. Dokl., 30:3 (1984), 588-591.

[27] А.П. Веселов, С. П. Новиков, "Конечнозонные двумерные операторы Шрёдингера. Потенциальные операторы", Докл. АН СССР, 279:4 (1984), 784-788; англ. пер.: A.P. Veselov, S. P. Novikov, "Finite-zone, two-dimensional Schrödinger operators. Potential operators", Soviet Math. Dokl., 30:3 (1984), 705-708.

[28] A. P. Veselov, I. M. Krichever, S. P. Novikov, "Two-dimensional periodic Schrödinger operators and Prym's $\theta$-functions", Geometry today (Rome, 1984), Progr. Math., 60, Birkhäuser Boston, Boston, MA, 1985, 283-301. 
[29] S. P. Novikov, A. P. Veselov, "Two-dimensional Schrödinger operator: inverse scattering transform and evolutional equations", Phys. D, 18:1-3 (1986), 267-273.

[30] П. Г. Гриневич, С. В. Манаков, "Обратная задача теории рассеяния для двумерного оператора Шрёдингера, $\bar{\partial}$-метод и нелинейные уравнения”, Функи. анализ и его прил., 20:2 (1986), 14-24; англ. пер.: P. G. Grinevich, S. V. Manakov, "Inverse scattering problem for the two-dimensional Schrödinger operator, the $\bar{\partial}$-method and nonlinear equations", Funct. Anal. Appl., 20:2 (1986), 94-103.

[31] П. Г. Гриневич, Р. Г. Новиков, "Аналоги многосолитонных потенциалов для двумерного оператора Шрёдингера", Функи. анализ и его прил., 19:4 (1985), 32-42; англ. пер.: P. G. Grinevich, R. G. Novikov, "Analogs of multisoliton potentials for the two-dimensional Schrödinger operator", Funct. Anal. Appl., 19:4 (1985), 276-285.

[32] П. Г. Гриневич, С. П. Новиков, “Двумерная 'обратная задача рассеяния' для отрицательных энергий и обобщенно-аналитические функции. I. Энергии ниже основного состояния", Функи. анализ и его прил., 22:1 (1988), 23-33; англ. пер.: P. G. Grinevich, S. P. Novikov, "Two-dimensional 'inverse scattering problem' for negative energies and generalized-analytic functions. I. Energies below the ground state", Funct. Anal. Appl., 22:1 (1988), 19-27.

[33] И. М. Кричевер, "Спектральная теория двумерных периодических операторов и ее приложения", УМH, 44:2(266) (1989), 121-184; англ. пер.: I. M. Krichever, "Spectral theory of two-dimensional periodic operators and its applications", Russian Math. Surveys, 44:2 (1989), 145-225.

[34] B. G. Konopel'chenko, "The two-dimensional second-order differential spectral problem: compatibility conditions, general BTs and integrable equations", Inverse Problems, 4:1 (1988), 151-163.

[35] И. М. Кричевер, “Эллиптические решения уравнения Кадомцева-Петвиашвили и интегрируемые системы частиц", Функи. анализ и его прил., 14:4 (1980), 45-54; англ. пер.: I. M. Krichever, "Elliptic solutions of the Kadomtsev-Petviashvili equation and integrable systems of particles", Funct. Anal. Appl., 14:4 (1980), 282-290.

\section{Петр Георгиевич Гриневич}

(Petr G. Grinevich)

Институт теоретической физики

им. Л. Д. Ландау РАН

E-mail: pgg@landau.ac.ru

\section{Андрей Евгеньевич Миронов \\ (Andrei E. Mironov)}

Институт математики им. С. Л. Соболева СО РАН;

Новосибирский государственный университет

E-mail: mironov@math.nsc.ru

\section{Сергей Петрович Новиков \\ (Sergey P. Novikov)}

University of Maryland, College Park, MD, USA;

Институт теоретической физики

им. Л. Д. Ландау РАН;

Математический институт им. В. А. Стеклова РАН

E-mail: novikov@ipst.umd.edu 